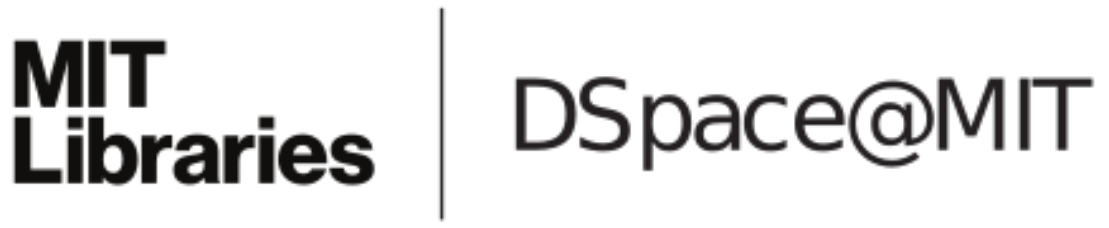

\author{
MIT Open Access Articles
}

\section{Efficient Routing Algorithms for Multiple Vehicles With no Explicit Communications}

The MIT Faculty has made this article openly available. Please share how this access benefits you. Your story matters.

Citation: Arsie, A., K. Savla, and E. Frazzoli. “Efficient Routing Algorithms for Multiple Vehicles With no Explicit Communications." Automatic Control, IEEE Transactions on 54.10 (2009): 2302-2317. (C) 2009 IEEE

As Published: http://dx.doi.org/10.1109/tac.2009.2028954

Publisher: Institute of Electrical and Electronics Engineers

Persistent URL: http://hdl.handle.net/1721.1/52393

Version: Final published version: final published article, as it appeared in a journal, conference proceedings, or other formally published context

Terms of Use: Article is made available in accordance with the publisher's policy and may be subject to US copyright law. Please refer to the publisher's site for terms of use. 


\title{
Efficient Routing Algorithms for Multiple Vehicles With no Explicit Communications
}

\author{
Alessandro Arsie, Ketan Savla, Student Member, IEEE, and Emilio Frazzoli, Senior Member, IEEE
}

\begin{abstract}
In this paper, we consider a class of dynamic vehicle routing problems, in which a number of mobile agents in the plane must visit target points generated over time by a stochastic process. It is desired to design motion coordination strategies in order to minimize the expected time between the appearance of a target point and the time it is visited by one of the agents. We propose control strategies that, while making minimal or no assumptions on communications between agents, provide the same level of steadystate performance achieved by the best known decentralized strategies. In other words, we demonstrate that inter-agent communication does not improve the efficiency of such systems, but merely affects the rate of convergence to the steady state. Furthermore, the proposed strategies do not rely on the knowledge of the details of the underlying stochastic process. Finally, we show that our proposed strategies yield an efficient, pure Nash equilibrium in a game theoretic formulation of the problem, in which each agent's objective is to maximize the expected value of the "time spent alone" at the next target location. Simulation results are presented and discussed.
\end{abstract}

Index Terms-Dynamic vehicle routing, facility location, multiagent systems, spatial games.

\section{INTRODUCTION}

A very active research area today addresses coordination of several mobile agents: groups of autonomous robots and large-scale mobile networks are being considered for a broad class of applications [1].

An area of particular interest is concerned with the generation of efficient cooperative strategies for several mobile agents to move through a certain number of given target points, possibly avoiding obstacles or threats [2]-[6]. Trajectory efficiency in these cases is understood in terms of cost for the agents: in other words, efficient trajectories minimize the total path length, the time needed to complete the task, or the fuel/energy expenditure. A related problem has been investigated as the Weapon-Target Assignment (WTA) problem, in which mobile agents are allowed to team up in order to enhance the probability of a favorable outcome in a target engagement [7], [8]. In this setup,

Manuscript received August 04, 2007; revised September 15, 2008 and January 02, 2009. First published September 22, 2009; current version published October 07, 2009. This was supported by National Science Foundation and Air Force Office of Scientific Research Grants 0325716 (NSF), 0715025 (NSF), 0705451 (NSF), 0705453 (NSF), and F49620-02-1-0325 (AFOSR). Recommended by Associate Editor J. Lygeros.

A. Arsie is with the Mathematics Department, Penn State University, University Park, PA 16802 USA (e-mail: arsie@ math.psu.edu).

K. Savla and E. Frazzoli are with the Laboratory for Information and Decision Systems, Massachusetts Institute of Technology, Cambridge, MA 02139 USA (e-mail:ksavla@mit.edu; frazzoli@mit.edu).

Color versions of one or more of the figures in this paper are available online at http://ieeexplore.ieee.org.

Digital Object Identifier 10.1109/TAC.2009.2028954 targets locations are known and an assignment strategy is sought that maximizes the global success rate. In a biological setting, the closest parallel to many of these problems is the development of foraging strategies, and of territorial versus gregarious behaviors [9], in which individuals choose to identify and possibly defend a hunting ground.

In this paper, we consider a class of cooperative motion coordination problems, which we can refer to as dynamic vehicle routing, in which service requests are not known a priori, but are dynamically generated over time by a stochastic process in a geographic region of interest. Each service request is associated to a target point in the plane, and is fulfilled when one of a team of mobile agents visits that point. For example, service requests can be thought of as threats to be investigated in a surveillance application, events to be measured in an environmental monitoring scenario, and as information packets to be picked up and delivered to a user in a wireless sensor network. It is desired to design a control strategy for the mobile agents that provably minimizes the expected waiting time between the issuance of a service request and its fulfillment. In other words, our focus is on the quality of service as perceived by the "end user," rather than, for example, fuel economies achieved by the mobile agents. Similar problems were also considered in [10], [11], and decentralized strategies were presented in [12]. This problem has connections to the Persistent Area Denial (PAD) and area coverage problems discussed, e.g., in [4], [13]-[15].

A common theme in cooperative control is the investigation of the effects of different communication and information sharing protocols on the system performance. Clearly, the ability to access more information at each single agent can not decrease the performance level; hence, it is commonly believed that by providing better communication among agents will improve the system's performance. In this paper, we prove that there are certain dynamic vehicle routing problems which can, in fact, be solved (almost) optimally without any explicit communication between agents; in other words, the no-communication constraint in such cases is not binding, and does not limit the steady-state performance. The main contribution of this paper is the introduction of a motion coordination strategy that does not require any explicit communication between agents, while achieving provably optimal performance in certain conditions.

The paper is structured as follows: in Section II we set up and formulate the problem we investigate in the paper. In Section III we introduce the proposed solution algorithms, and discuss their characteristics. Section IV is the technical core of the paper, in which we prove the convergence of the performance provided by the proposed algorithms to a critical point (either 
a local minimum or a saddle point) of the global performance function. Moreover, we show that any optimal configuration corresponds to a class of tessellations of the plane that we call Median Voronoi Tessellations. Section V is devoted to a game-theoretic interpretation of our results in which the agents are modeled as rational autonomous decision makers trying to maximize their own utility function. We prove that, our algorithms yield an efficient, pure Nash equilibrium in a game theoretic formulation of the problem. In Section VI we present some numerical results, while Section VII is dedicated to final remarks and further extensions of this line of research.

\section{PROBlem Formulation}

Let $\Omega \subset \mathbb{R}^{2}$ be a convex domain on the plane, with nonempty interior; we will refer to $\Omega$ as the workspace. A stochastic process generates service requests over time, which are associated to points in $\Omega$; these points are also called targets. The process generating service requests is modeled as a spatio-temporal Poisson point process, with temporal intensity $\lambda>0$, and an absolutely continuous spatial distribution described by the density function $\varphi: \Omega \rightarrow \mathbb{R}_{+}$, with bounded and convex support within $\Omega$ (i.e., $\varphi(q)>0 \Leftrightarrow q \in \mathcal{Q} \subseteq \Omega$, with $\mathcal{Q}$ bounded and convex). The spatial density function $\varphi$ is normalized in such a way that $\int_{\Omega} \varphi(q) d q=1$. Both $\lambda$ and $\varphi$ are not necessarily known.

A spatio-temporal Poisson point process is a collection of functions $\left\{\mathcal{P}: \overline{\mathbb{R}}_{+} \rightarrow 2^{\Omega}\right\}$ such that, for any $t>0, \mathcal{P}(t)$ is a random collection of points in $\Omega$, representing the service requests generated in the time interval $[0, t)$, and such that

- The total numbers of events generated in two disjoint timespace regions are independent random variables;

- The total number of events occurring in an interval $[s, s+t)$ in a measurable set $\mathcal{S} \subseteq \Omega$ satisfies

$$
\begin{aligned}
\operatorname{Pr}[\operatorname{card}((\mathcal{P}(s+t) & -\mathcal{P}(s)) \cap \mathcal{S})=k] \\
= & \frac{\exp (-\lambda t \cdot \varphi(\mathcal{S}))(\lambda t \cdot \varphi(\mathcal{S}))^{k}}{k !}, \quad \forall k \in \mathbb{N}
\end{aligned}
$$

where $\varphi(\mathcal{S})$ is a shorthand for $\int_{\mathcal{S}} \varphi(q) d q$.

Each particular function $\mathcal{P}$ is a realization, or trajectory, of the Poisson point process. A consequence of the properties defining Poisson processes is that the expected number of targets generated in a measurable region $S \subseteq \Omega$ during a time interval of length $\Delta t$ is given by

$$
\mathrm{E}[\operatorname{card}((\mathcal{P}(t+\Delta t)-\mathcal{P}(t)) \cap \mathcal{S})]=\lambda \Delta t \cdot \varphi(\mathcal{S}) .
$$

Without loss of generality, we will identify service requests with targets points, and label them in order of generation; in other words, given two targets $e_{i}, e_{j} \in \mathcal{P}(t)$, with $i<j$, the service request associated with these target have been issued at times $t_{i} \leq t_{j} \leq t$ (since events are almost never generated concurrently, the inequalities are in fact strict almost surely).

A service request is fulfilled when one of $m$ mobile agents, modeled as point masses, moves to the target point associated with it; $m$ is a possibly large, but finite number. Let $p(t)=$ $\left(p_{1}(t), p_{2}(t), \ldots, p_{m}(t)\right) \in \Omega^{m}$ be a vector describing the positions of the agents at time $t$. (We will tacitly use a similar notation throughout the paper). The agents are free to move, with bounded speed, within the workspace $\Omega$; without loss of generality, we will assume that the maximum speed is unitary. In other words, the dynamics of the agents are described by differential equations of the form

$$
\frac{d p_{i}(t)}{d t}=u_{i}(t), \quad \text { with } \quad\left\|u_{i}(t)\right\| \leq 1, \quad \forall t \geq 0
$$

for each $i \in\{1, \ldots, m\}$. The agents are identical, and have unlimited range and target-servicing capability.

Let $\mathcal{B}_{i}(t) \subset \Omega$ indicate the set of targets serviced by the $i$-th agent up to time $t$. (By convention, $\mathcal{B}_{i}(0)=\emptyset, i=1, \ldots, m$ ). We will assume that $\mathcal{B}_{i} \cap \mathcal{B}_{j}=\emptyset$ if $i \neq j$, i.e., that service requests are fulfilled by at most one agent. (In the unlikely event that two or more agents visit a target at the same time, the target is arbitrarily assigned to one of them).

Let $\mathcal{D}: t \rightarrow 2^{\Omega}$ indicate (a realization of) the stochastic process obtained by combining the service request generation process $\mathcal{P}$ and the removal process caused by the agents servicing outstanding requests; in other words

$$
\begin{aligned}
\mathcal{P}(t) & =\mathcal{D}(t) \cup \mathcal{B}_{1}(t) \cup \cdots \cup \mathcal{B}_{m}(t) \\
\mathcal{D}(t) \cap \mathcal{B}_{i}(t) & =\emptyset
\end{aligned}
$$

for each $i \in\{1, \ldots, m\}$. The random set $\mathcal{D}(t) \subset \Omega$ represents the demand, i.e., the service requests outstanding at time $t$; let $n(t):=\operatorname{card}(\mathcal{D}(t))$.

In this paper we will concentrate on motion coordination strategies of the following two forms:

$$
\pi_{i}:\left(p_{i}, \mathcal{B}_{i}, \mathcal{D}\right) \mapsto u_{i}, \quad i \in\{1, \ldots, m\}
$$

and

$$
\pi_{i}:\left(p_{1}, \ldots, p_{m}, \mathcal{B}_{i}, \mathcal{D}\right) \mapsto u_{i}, \quad i \in\{1, \ldots, m\} .
$$

An agent executing a control policy of the form (2) relies on the knowledge of its own current position, on a record of targets it has previously visited, and on the current demand. In other words, such control policies do not need any explicit information exchange between agents; as such, we will refer to them as no communication (nc) policies. Such policies are trivially decentralized.

Following the first control policy, it is certainly true that agent $i$ must know the set $\mathcal{D}(t)$ of outstanding targets, but this does not mean that this agent must know any of the sets $\mathcal{B}_{j}(t)$, for $j \neq i$. It is true that agents implicitly share/update the set of tasks that must be completed, but this does not mean that there is any communication among the agents. This is how the agents can have complete knowledge of $\mathcal{D}(t)$ without any communication among themselves (note that it is possible to find out other ways to achieve a complete knowledge of $\mathcal{D}(t)$ without communication, what follows is simply one of the possible ways). Indeed, suppose that the targets broadcast their service requests and their positions to the whole environment and they immediately stop broadcasting as soon as they are visited by one 
of the agents. Suppose also that each agent is able to receive the signal broad-casted by each target and is able to detect the corresponding target position. In this set-up each agent knows precisely what are the outstanding targets, without exchanging directly any information with other agents. On the other hand, each agent seeing that a target has stopped broadcasting its service request can infer that that particular target has been visited. This is however not a communication among the agents, but simply an inference. The no-communication hypothesis is referred to the lack of direct communication among the agents, not to the inferences that each single agent can perform observing how the service requests of the outstanding targets are satisfied.

On the other hand, an agent executing a control policy of the form (3) can sense the current position of other agents, but still has information only on the targets itself visited in the past. We call these sensor-based (sb) policies, to signify the fact that only factual information is exchanged between agents-as opposed to information related to intent and past history. Note that both the families of coordination policies rely, in principle, on the knowledge of the locations of all outstanding targets. However only local target sensing will be necessary in practice. This last claim is difficult to justify from a theoretical point of view, and it is better understood in terms of simulations. For a more complete treatment of this issue, in particular the effect of limiting sensing radius for target sensing and its effect on the system performance, see the review [16].

A policy $\pi=\left(\pi_{1}, \pi_{2}, \ldots, \pi_{m}\right)$ is said to be stabilizing if, under its effect, the expected number of outstanding targets does not diverge over time, i.e., if

$$
\begin{aligned}
\bar{n}_{\pi} & :=\lim _{t \rightarrow+\infty} \mathrm{E}\left[n(t) \mid \dot{p}_{i}(t)\right. \\
& \left.=\pi_{i}\left(p(t), \mathcal{B}_{i}(t), \mathcal{D}(t)\right), i \in\{1, \ldots, m\}\right]
\end{aligned}
$$

is finite. Intuitively, a policy is stabilizing if the mobile agents are able to visit targets at a rate that is —on average — at least as fast as the rate at which new service requests are generated.

Let $T_{j}$ be the time elapsed between the issuance of the $j$-th service request, and the time it is fulfilled. If the system is stable, then the following balance equation (also known as Little's formula [17]) holds:

$$
\bar{n}_{\pi}=\lambda \bar{T}_{\pi}
$$

where $\bar{T}_{\pi}:=\lim _{j} \rightarrow \infty \mathrm{E}\left[T_{j}\right]$ is the system time under policy $\pi$, i.e., the expected time a service request must wait before being fulfilled, given that the mobile agents follow the strategy defined by $\pi$. Note that the system time $\bar{T}_{\pi}$ can be thought of as a measure of the quality of service, as perceived by the "user" issuing the service requests.

At this point we can finally state our problem: we wish to devise a policy that is (i) stabilizing, and (ii) yields a quality of service (i.e., system time) achieving, or approximating, the theoretical optimal performance given by

$$
\bar{T}_{\text {opt }}=\inf _{\pi \text { stabilizing }} \bar{T}_{\pi} .
$$

Centralized and decentralized strategies are known that optimize or approximate (5) in a variety of cases of interest [11],

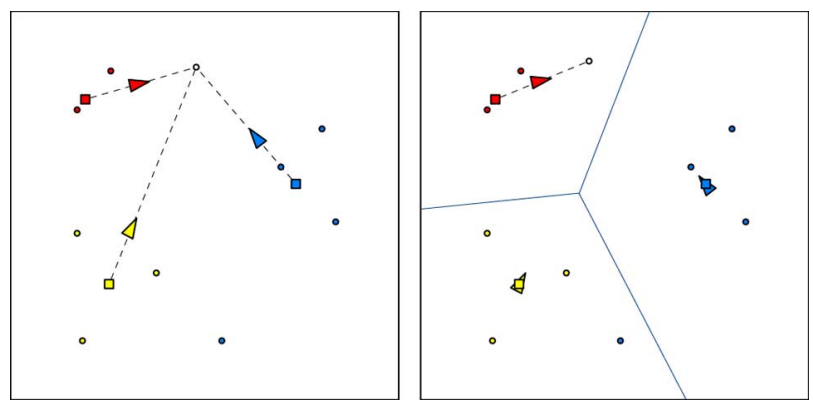

Fig. 1. Illustration of the two control policies proposed in Section III. While no targets are outstanding, vehicles wait at the point that minimizes the average distance to targets they have visited in the past; such points are depicted as squares, while targets are circles and vehicles triangles. In the no-communication policy, at the appearance of a new target, all vehicles pursue it (left). In the sensor-based policy, only the vehicle that is closest to the target will pursue it (right).

[12], [18], [19]. However, all such strategies rely either on a central authority with the ability to communicate to all agents, or on the exchange of certain information about each agent's strategy with other neighboring agents. In addition, these policies require the knowledge of the spatial distribution $\varphi$; decentralized versions of these implement versions of Lloyd's algorithm for vector quantization [20].

In the remainder of this paper, we will investigate how the additional constraints posed on the exchange of information between agents by the models (2) and (3) impact the achievable performance and quality of service. Remarkably, the policies we will present do not rely on the knowledge of the spatial distribution $\varphi$, and are a generalized version of MacQueen's clustering algorithm [21].

\section{CONTROL POLICY DESCRIPTION}

In this section, we introduce two control policies of the forms, respectively, (2) and (3). An illustration of the two policies is given in Fig. 1.

\section{A. A Control Policy Requiring no Explicit Communication}

Let us begin with an informal description of a policy $\pi_{\mathrm{nc}}$ requiring no explicit information exchange between agents. At any given time $t$, each agent computes its own control input according to the following rule:

1) If $\mathcal{D}(t)$ is not empty, move towards the nearest outstanding target.

2) If $\mathcal{D}(t)$ is empty, move towards the point minimizing the average distance to targets serviced in the past by each agent. If there is no unique minimizer, then move to the nearest one.

In other words, we set

$$
\pi_{\mathrm{nc}}\left(p_{i}(t), \mathcal{B}_{i}(t), \mathcal{D}(t)\right)=\operatorname{vers}\left(F_{\mathrm{nc}}\left(p_{i}(t), \mathcal{B}_{i}(t), \mathcal{D}(t)\right)-p_{i}(t)\right)
$$

where

$$
F_{\mathrm{nc}}\left(p_{i}, \mathcal{B}_{i}, \mathcal{D}\right)= \begin{cases}\arg \min _{q \in \mathcal{D}}\left\|p_{i}-q\right\|, & \text { if } \mathcal{D} \neq \emptyset, \\ \arg \min _{q \in \Omega} \sum_{e \in \mathcal{B}_{i}}\|e-q\|, & \text { otherwise }\end{cases}
$$


$\|\cdot\|$ is the Euclidean norm, and

$$
\operatorname{vers}(v)= \begin{cases}\frac{v}{\|v\|,}, & \text { if } v \neq 0 \\ 0 & \text { otherwise. }\end{cases}
$$

The convex function $W: q \mapsto \sum_{e \in \mathcal{B}}\|q-e\|$, often called the (discrete) Weber function in the facility location literature [22], [23] (modulo normalization by $\operatorname{card}(\mathcal{B})$ ), is not strictly convex only when the point set $\mathcal{B}$ is empty-in which case we set $W(\cdot)=0$ by convention - or contains an even number of collinear points. In such cases, the minimizer nearest to $p_{i}$ in (7) is chosen. We will call the point $p_{i}^{*}(t)=F_{\mathrm{nc}}\left(\cdot, \mathcal{B}_{i}(t), \emptyset\right)$ the reference point for the $i$-th agent at time $t$.

In the $\pi_{\mathrm{nc}}$ policy, whenever one or more service requests are outstanding, all agents will be pursuing a target; in particular, when only one service request is outstanding, all agents will move towards it. When the demand queue is empty, agents will either i) stop at the current location, if they have visited no targets yet, or ii) move to their reference point, as determined by the set of targets previously visited.

\section{B. A Sensor-Based Control Policy}

The control strategy in the previous section can be modified to include information on the current position of other agents, if available (e.g., through on-board sensors). In order to present the new policy, indicate with $\mathcal{V}(p)=\left\{\mathcal{V}_{1}(p), \mathcal{V}_{2}(p), \ldots, \mathcal{V}_{m}(p)\right\}$ the Voronoi partition of the workspace $\Omega$, defined as:

$$
\mathcal{V}_{i}(p)=\left\{q \in \Omega:\left\|q-p_{i}\right\| \leq\left\|q-p_{j}\right\|, \forall j=1 \ldots m\right\} .
$$

As long as an agent has never visited any target, i.e., as long as $\mathcal{B}_{i}(t)=\emptyset$, it executes the $\pi_{\mathrm{nc}}$ policy. Once an agent has visited at least one target, it computes its own control input according to the following rule:

1) If $\mathcal{D}(t) \cap \mathcal{V}_{i}(t)$ is not empty, move towards the nearest outstanding target in the agent's own Voronoi region.

2) If $\mathcal{D}(t) \cap \mathcal{V}_{i}(t)$ is empty, move towards the point minimizing the average distance to targets in $\mathcal{B}_{i}(t)$. If there is no unique minimizer, then move to the nearest one.

In other words, we set

$$
\pi_{\mathrm{sb}}\left(p(t), \mathcal{B}_{i}(t), \mathcal{D}(t)\right)=\operatorname{vers}\left(F_{\mathrm{sb}}\left(p(t), \mathcal{B}_{i}(t), \mathcal{D}(t)\right)-p_{i}(t)\right)
$$

where

$$
\begin{aligned}
& F_{\mathrm{sb}}\left(p, \mathcal{B}_{i}, \mathcal{D}\right) \\
& = \begin{cases}\arg \min _{q \in \mathcal{D}}\left\|p_{i}-q\right\|, & \text { if } \mathcal{D} \neq \emptyset, \text { and } \mathcal{B}_{i}=\emptyset \\
\arg \min _{q \in \mathcal{D} \cap \mathcal{V}_{i}(p)}\left\|p_{i}-q\right\|, & \text { if } \mathcal{D} \cap \mathcal{V}_{i} \neq \emptyset, \text { and } \mathcal{B}_{i} \neq \emptyset \\
\arg \min _{q \in \Omega} \sum_{e \in \mathcal{B}_{i}}\|e-q\|, & \text { otherwise. }\end{cases}
\end{aligned}
$$

In the $\pi_{\mathrm{sb}}$ policy, at most one agent will be pursuing a given target, at any time after an initial transient that terminates when all agents have visited at least one target each. The agents' behavior when no outstanding targets are available in their Voronoi region is similar to that determined by the $\pi_{\mathrm{nc}}$ policy previously discussed, i.e., they move to their reference point, determined by previously visited targets.

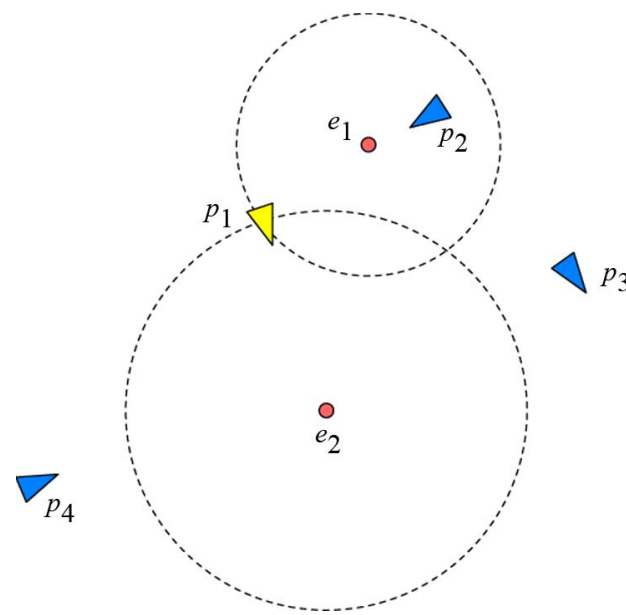

Fig. 2. Implicit computation of Voronoi regions: Even though target $e_{1}$ is the nearest target to $p_{1}$, it is not in the Voronoi region of the 1 st agent. In fact, the circle of radius $\left\|e_{1}-p_{1}\right\|$ centered at $e_{1}$ contains $p_{2}$, and the 2 nd agent is closer to $e_{1}$. However, the circle of radius $\left\|e_{2}-p_{1}\right\|$ centered at $e_{2}$ does not contain any other agent, ensuring that $e_{2}$ is in the Voronoi region generated by $p_{1}$.

Remark 1: While we introduced Voronoi partitions in the definition of the control policy, the explicit computation of each agent's Voronoi region is not necessary. In fact, each agent only needs to check whether it is the closest agent to a given target or not. In order to check whether a target point $q$ is in the Voronoi region of the $i$-th agent, it is necessary to know the current position only of agents within a circle or radius $\left\|p_{i}-q\right\|$ centered at $q$ (see Fig. 2). For example, if such circle is empty, then $q$ is certainly in $\mathcal{V}_{i}$; if the circle is not empty, distances of the agents within it to the target must be compared. This provides a degree of spatial decentralization - with respect to other agents - that is even stronger than that provided by restricting communications to agents sharing a boundary in a Voronoi partition [i.e., neighboring agents in the Delaunay graph, dual to the partition (8)].

Remark 2: The sensor-based policy is more efficient than the no-communication policy in terms of the length of the path traveled by each agent, since there is no duplication of effort as several agents pursue the same target (unless a target is on the boundary of two or more Voronoi regions). However, in terms of "quality of service," we will show that there is no difference between the two policies, for low target generation rates. $\mathrm{Nu}$ merical results show that the sensor-based policy is more efficient in a broader range of target generation rates, and in fact provides almost optimal performance both in light and heavy load conditions.

\section{Performance Analysis in Light LoAD}

In this section we analyze the performance of the control policies proposed in the previous section. In particular, we concentrate our investigation on the light load case, in which the target generation rate is very small, i.e., as $\lambda \rightarrow 0^{+}$. This will allow us to prove analytically certain interesting and perhaps surprising characteristics of the proposed policies. The performance analysis in the general case is more difficult; we will discuss the results of numerical investigation in Section VI, but no analytical results are available at this time. 


\section{A. Overview of the System Behavior in the Light Load Regime}

Before starting a formal analysis, let us summarize the key characteristics of the agents' behavior in light load, i.e., for small values of $\lambda$.

1) At the initial time the $m$ agents are assumed to be deployed at general positions in $\Omega$, and the demand queue is empty, $\mathcal{D}(0)=\emptyset$.

2) The agents do not move until the first service request appears. At that time, if the policy $\pi_{\mathrm{nc}}$ is used, all agents will start moving towards the first target. If the sensor-based policy $\pi_{\mathrm{sb}}$ is used, only the closest agent will move towards the target.

3) As soon as one agent reaches the target, all agents start moving towards their current reference point, and the process continues.

For small $\lambda$, with high probability (i.e., with probability approaching 1 as $\lambda \rightarrow 0^{+}$) at most one service request is outstanding at any given time. In other words, new service requests are generated so rarely that most of the time agents will be able to reach a target and return to their reference point before a new service request is issued. This is just the content of the following.

Proposition 3: Each agent will be able to return to its reference point before the generation of a new service request infinitely often with high probability as $\lambda \rightarrow 0^{+}$.

Proof: Let $t_{1}$ be such that $\mathcal{B}_{i}\left(t_{1}\right) \neq \emptyset$, for all $i \in\{1, \ldots, m\}$. Such a time exists, almost surely, basically because of the fairness of the proposed policies. At time $t_{1}$ all agents will be within $\mathcal{Q}$. Let $n_{1}=\operatorname{card}\left(\mathcal{D}\left(t_{1}\right)\right)$ be the total number of outstanding targets at time $t_{1}$. An upper bound on the time needed to visit all targets in $\mathcal{D}\left(t_{1}\right)$ is $n_{1}(\operatorname{diam}(\mathcal{Q}))$. When there are no outstanding targets, agents move to their reference points, reaching them in at most $\operatorname{diam}(\mathcal{Q})$ units of time.

The time needed to service the initial targets and go to the reference configuration is hence bounded by $t_{\text {ini }} \leq t_{1}+\left(n_{1}+\right.$ $1) \operatorname{diam}(\mathcal{Q})$. The probability that at the end of this initial phase the number of targets is reduced to zero is

$$
\begin{aligned}
P\left[n\left(t_{\text {ini }}\right)=0\right] & =\exp \left(-\lambda\left(t_{\text {ini }}-t_{1}\right)\right) \\
& \geq \exp \left(-\lambda\left(n_{1}+1\right) \operatorname{diam}(\mathcal{Q})\right)
\end{aligned}
$$

that is, $P\left[n\left(t_{\text {ini }}\right)=0\right] \rightarrow 1^{-}$as $\lambda \rightarrow 0^{+}$. As a consequence, after an initial transient, all targets will be generated with all agents waiting at their reference points, and an empty demand queue, with high probability as $\lambda \rightarrow 0^{+}$.

Consider the $j$-th service request, generated at time $t_{j}$. Assuming that, at $t_{j}$, all agents are at their reference positions, the expected system time $\mathrm{E}\left[T_{j}\right]$ can be computed as

$$
\mathrm{E}\left[T_{j}\right]=\int_{\Omega} \min _{i=1, \ldots, m}\left\|p_{i}^{*}\left(t_{j}\right)-q\right\| \varphi(q) d q .
$$

Assume for now that the sequences $\left\{p_{i}^{*}\left(t_{j}\right): j \in \mathbb{N}\right\}$ converge, and let

$$
\lim _{j \rightarrow+\infty} p_{i}^{*}\left(t_{j}\right)=\hat{p}_{i}^{*}
$$

Note that $\hat{p}_{i}^{*}$ is a random variable, the value of which depends in general on the particular realization of the target generation process. If all service requests are generated with the agents at their reference positions, the average service time (for small $\lambda$ ) can be evaluated as

$$
\begin{aligned}
\bar{T}_{\mathrm{nc}}=\bar{T}_{\mathrm{sb}} & =\int_{\Omega} \min _{i=1, \ldots, m}\left\|\hat{p}_{i}^{*}-q\right\| \varphi(q) d q \\
& =\sum_{i=1}^{m} \int_{\mathcal{V}_{i}\left(p^{*}\right)}\left\|\hat{p}_{i}^{*}-q\right\| \varphi(q) d q .
\end{aligned}
$$

Since the system time depends on the random variable $\hat{p}^{*}=$ $\left(\hat{p}_{1}^{*}, \ldots, \hat{p}_{m}^{*}\right)$, it is itself a random variable. The function appearing on the right hand side of the above equation, relating the system time to the asymptotic location of reference points, is called the continuous multi-median function [22]. This function admits a global minimum (in general not unique) for all non-singular density functions $\varphi$, and in fact it is known [11] that the optimal performance in terms of system time is given by

$$
\bar{T}_{\mathrm{opt}}=\min _{p \in \Omega^{m}} \sum_{i=1}^{m} \int_{\mathcal{V}_{i}(p)}\left\|p_{i}-q\right\| \varphi(q) d q .
$$

In the following, we will investigate the convergence of the reference points as new targets are generated, in order to draw conclusions about the average system time $\bar{T}$ in light load. In particular, we will prove not only that the reference points converge with high probability (as $\lambda \rightarrow 0^{+}$) to a local critical point (more precisely, either local minima or saddle points) for the average system time, but also that the limiting reference points $\hat{p}^{*}$ are generalized medians of their respective Voronoi regions, where

Definition 4 (Generalized Median): The generalized median of a set $\mathcal{S} \subset \mathbb{R}^{n}$ with respect to a density function $\varphi: \mathcal{S} \rightarrow \overline{\mathbb{R}}_{+}$ is defined as

$$
\bar{p}:=\arg \min _{p \in \mathbb{R}^{n}} \int_{\mathcal{S}}\|p-q\| \varphi(q) d q .
$$

We call the resulting Voronoi tessellation Median Voronoi Tessellation (MVT for short), in analogy with what is done with Centroidal Voronoi Tessellations. A formal definition is as follows:

Definition 5 (Median Voronoi Tessellation): A Voronoi tessellation $\mathcal{V}(p)=\left\{\mathcal{V}_{1}(p), \ldots, \mathcal{V}_{m}(p)\right\}$ of a set $\mathcal{S} \subset \mathbb{R}^{n}$ is called a Median Voronoi Tessellation of $\mathcal{S}$ with respect to the density function $\varphi$ if the ordered set of generators $p$ is equal to the ordered set of generalized medians of the sets in $\mathcal{V}(p)$ with respect to $\varphi$, i.e., if

$$
p_{i}=\arg \min _{s \in \mathbb{R}^{n}} \int_{\mathcal{V}_{i}(p)}\|s-q\| \varphi(q) d q, \quad \forall i \in\{1, \ldots, m\} .
$$

Since the proof builds on a number of intermediate results, we provide an outline of the argument as a convenience to the reader.

1) First, we prove that the reference point of any agent that visits an unbounded number of targets over time converges almost surely.

2) Second, we prove that, if $m \geq 1$ agents visit an unbounded number of targets over time, their reference points will converge to the generators of a MVT almost surely, as long as agents are able to return to their reference point infinitely often. 
3) Third, we prove that all agents will visit an unbounded number of targets (this corresponds to a property of distributed algorithms that is often called fairness in computer science).

4) Finally, we prove that agents are able to return to their reference points infinitely often with high probability as $\lambda \rightarrow 0^{+}$.

Combining these steps, together with (5), will allow us to state that the reference points converge to a local critical point of the system time, with high probability as $\lambda \rightarrow 0^{+}$.

\section{B. Convergence of Reference Points}

Let us consider an agent $i$, such that

$$
\lim _{t \rightarrow+\infty} \operatorname{card}\left(\mathcal{B}_{i}(t)\right)=+\infty
$$

i.e., an agent that services an unbounded number of requests over time. Since the number of agents $m$ is finite, and the expected number of targets generated over a time interval $[0, t)$ is proportional to $t$, at least one such agent will always exist. In the remainder of this section, we will drop the subscript $i$, since we will consider only this agent, effectively ignoring all others for the time being.

For any finite $t$, the set $\mathcal{B}(t)$ will contain a finite number of points. Assuming that $\mathcal{B}(t)$ contains at least three non-collinear points, the discrete Weber function $p \mapsto \sum_{q \in \mathcal{B}(t)}\|p-q\|$ is strictly convex, and has a unique optimizer $p^{*}(t)=$ $\arg \min _{p \in \Omega} \sum_{q \in \mathcal{B}(t)}\|p-q\|$. The optimal point $p^{*}(t)$ is called the Fermat-Torricelli (FT) point—or the Weber point in the location optimization literature-associated with the set $\mathcal{B}(t)$; see [23]-[25] for a historical review of the problem and for solution algorithms.

It is known that the FT point is unique and algebraic for any set of non-collinear points. While there are no general analytic solutions for the location of the FT point associated to more than 4 points, numerical solutions can be easily constructed relying on the convexity of the Weber function, and on the fact that it is differentiable for all points not in $\mathcal{B}$. Polynomial-time approximation algorithms are also available (see, e.g., [26], [27]). Remarkably, a simple mechanical device can be constructed to solve the problem, based on the so-called Varignon frame, as follows. Holes are drilled on a horizontal board, at locations corresponding to the points in $\mathcal{B}$. A string attached to a unit mass is passed through each of these holes, and all strings are tied together at one end. The point reached by the knot at equilibrium is a FT point for $\mathcal{B}$.

Some useful properties of FT points are summarized below. If there is a $q_{0} \in \mathcal{B}$ such that

$$
\left\|\sum_{q \in \mathcal{B} \backslash q_{0}} \operatorname{vers}\left(q_{0}-q\right)\right\| \leq 1
$$

then $p^{*}=q_{0}$ is a FT point for $\mathcal{B}$. If no point in $\mathcal{B}$ satisfies such condition, then the FT point $p^{*}$ can be found as a solution of the following equation:

$$
\sum_{q \in \mathcal{B}} \operatorname{vers}\left(p^{*}-q\right)=0
$$

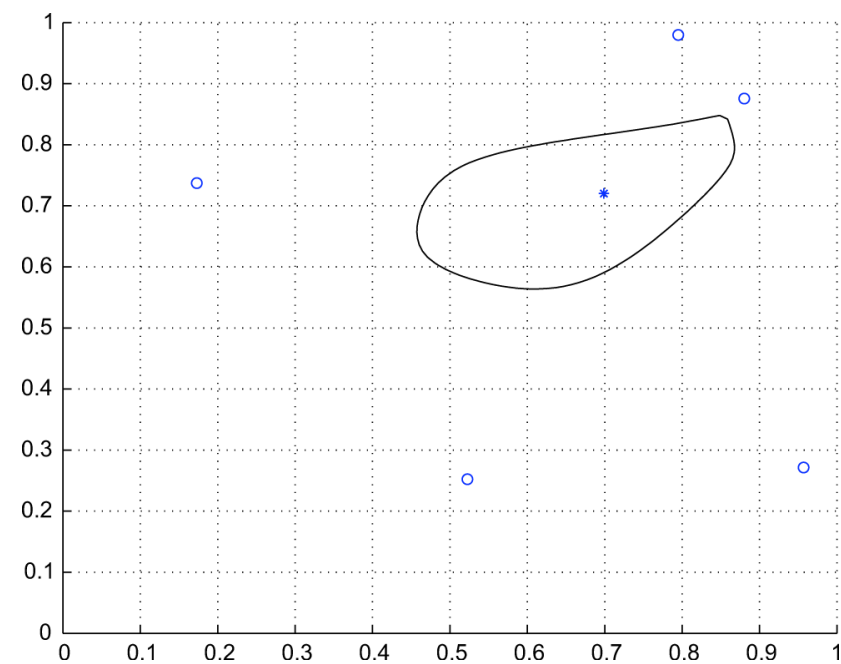

Fig. 3. Example of a Fermat-Torricelli point (star) and solution set corresponding to five target points (circles). Upon the addition of an arbitrarily chosen sixth target point, the Fermat-Torricelli is guaranteed to remain within the region bounded by the curve.

In other words, $p^{*}$ is simply the point in the plane at which the sum of unit vectors starting from it and directed to each of the points in $\mathcal{B}$ is equal to zero; this point is unique if $\mathcal{B}$ contains non-collinear points. Clearly, the FT point is in the convex hull of $\mathcal{B}$.

Note that the points in $\mathcal{B}(t)$ are randomly sampled from an unknown absolutely continuous distribution, described by a spatial density function $\tilde{\varphi}$ - which is not necessarily the same as $\varphi$, and in general is time-varying, depending on the past actions of all agents in the system. Even though $\tilde{\varphi}$ is not known, it can be expressed as

$$
\tilde{\varphi}(q, t)= \begin{cases}\frac{\varphi(q)}{\int_{\mathcal{I}(t)} \varphi(q) d q} & \text { if } q \in \mathcal{I}(t) \\ 0 & \text { otherwise }\end{cases}
$$

for some convex set $\mathcal{I}(t)$ containing $p(t)$. (In practical terms, such set will be the Voronoi region generated by $p(t)$, in any case this remark is given as an aside and it is not important in the analysis of the proposed policies).

The function $t \mapsto p^{*}(t)$ is piecewise constant, i.e., it changes value at the discrete time instants $\left\{t_{j}: j \in \mathbb{N}\right\}$ at which the agent visits new targets. As a consequence, we can concentrate on the sequence $\left\{p^{*}\left(t_{j}\right): j \in \mathbb{N}\right\}$, and study its convergence.

Definition 6: For any $t>0$, let the solution set $C(t)$ be defined as

$$
C(t):=\left\{p \in \Omega:\left\|\sum_{q \in \mathcal{B}(t)} \operatorname{vers}(p-q)\right\| \leq 1\right\} .
$$

An example of such set is shown in Fig. 3. Before exploring the properties of these sets, let us define $e_{j}$ the target point corresponding to the $j$-th service request. The reason for introducing such solution sets is that they have quite remarkable properties as shown by the following result.

Proposition 7: For any $j \in \mathbb{N}, p^{*}\left(t_{j+1}\right) \in C\left(t_{j}\right)$. More specifically, if $e_{j+1} \notin C\left(t_{j}\right)$ (i.e., the target point associated to the $j$-th service request is outside the solution set) then the FT point $p^{*}\left(t_{j+1}\right)$ is on the boundary of $C\left(t_{j}\right)$. If $e_{j+1} \in C\left(t_{j}\right)$, then $p^{*}\left(t_{j+1}\right)=e_{j+1}$. 


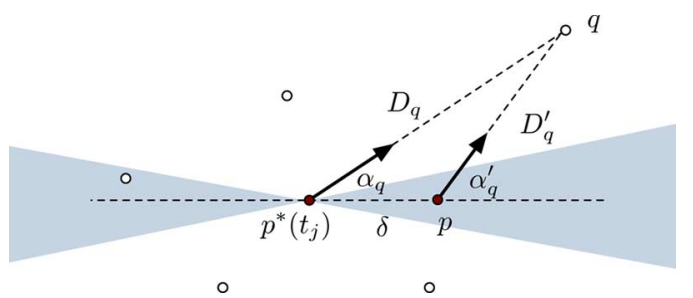

Fig. 4. Geometric constructions in the proof of Proposition 9.

Proof: If $e_{j+1}$ lies outside $C\left(t_{j}\right)$, we search for $p^{*}\left(t_{j+1}\right)$ as the solution of the equation

$$
\sum_{q \in \mathcal{B}\left(t_{j}\right)} \operatorname{vers}(p-q)+\operatorname{vers}\left(p-e_{j+1}\right)=0
$$

from which it turns out immediately

$$
\left\|\sum_{q \in \mathcal{B}\left(t_{j}\right)} \operatorname{vers}(p-q)\right\|=\left\|-\operatorname{vers}\left(p-e_{j+1}\right)\right\|=1
$$

thus $p^{*}\left(t_{j+1}\right) \in \partial C\left(t_{j}\right)$. Notice that is is not true in general that the solution $p^{*}\left(t_{j+1}\right)$ will lie on the line connecting $p^{*}\left(t_{j}\right)$ with the new target $e_{j+1}$. In the other case, if $e_{j+1}$ lies in $C\left(t_{j}\right)$, then it satisfies condition (13), and is the new FT point.

Now, in order to prove that the $\left\{p^{*}\left(t_{j}\right)\right\}_{j \in \mathbb{N}}$ converges to a point $\hat{p}^{*}$, we will prove that the diameter of the solution set $C\left(t_{j}\right)$ vanishes almost surely as $j$ tends to infinity. First we need the following result.

Proposition 8: If $\mathcal{Q}=\operatorname{Supp}(\varphi)$ is convex with non-empty interior, then $p^{*}(t) \in \operatorname{int}(\mathcal{Q})$ almost surely, for all $t$ such that $\mathcal{B}(t) \neq \emptyset$.

Proof: For any non-empty target set $\mathcal{B}(t)$, the FT point lies within the convex hull of $\mathcal{B}(t)$. All points in the set $\mathcal{B}(t)$ are contained within the interior of $\mathcal{Q}$ with probability one, since the boundary of $\mathcal{Q}$ is a set of measure zero. Since $\operatorname{int}(\mathcal{Q})$ is convex, and $\mathcal{B}(t) \subset \operatorname{int}(\mathcal{Q})$ almost surely, $p^{*}(t) \in \operatorname{co}(\mathcal{B}(t)) \subset \operatorname{int}(\mathcal{Q})$, almost surely.

Proposition 9: If the support of $\varphi$ is convex and bounded

$$
\lim _{j \rightarrow+\infty} \operatorname{diam}\left(C\left(t_{j}\right)\right)=0, \quad \text { a.s. }
$$

Proof: Consider a generic point $p \in C\left(t_{j}\right)$, and let $\delta=$ $p-p^{*}\left(t_{j}\right), \alpha_{q}=\arccos \left[\operatorname{vers}\left(p-p^{*}\left(t_{j}\right)\right) \cdot \operatorname{vers}\left(q-p^{*}\left(t_{j}\right)\right)\right]$, and $\alpha_{q}^{\prime}=\arccos \left[\operatorname{vers}\left(p-p^{*}\left(t_{j}\right)\right) \cdot \operatorname{vers}(q-p)\right]$, see Fig. 4 .

Since $p \in C\left(t_{j}\right)$, the magnitude of the sum of unit vectors $\sum_{q \in \mathcal{B}\left(t_{j}\right)} \operatorname{vers}(p-q)$ is no more than one, and the following inequality is true:

$$
\begin{aligned}
& \left|\left(\sum_{q \in \mathcal{B}\left(t_{j}\right)} \operatorname{vers}(p-q)\right) \cdot \operatorname{vers}\left(p-p^{*}\left(t_{j}\right)\right)\right| \\
& =\left|\sum_{q \in \mathcal{B}\left(t_{j}\right)}\left(\operatorname{vers}(p-q) \cdot \operatorname{vers}\left(p-p^{*}\left(t_{j}\right)\right)\right)\right| \\
& =\left|\sum_{q \in \mathcal{B}\left(t_{j}\right)} \cos \left(\alpha_{q}^{\prime}\right)\right| \leq 1 .
\end{aligned}
$$

Using elementary planar geometry, we obtain that

$$
\alpha_{q}^{\prime}-\alpha_{q} \geq \sin \left(\alpha_{q}^{\prime}-\alpha_{q}\right)=\frac{\delta \sin \left(\alpha_{q}\right)}{\|q-p\|} .
$$

Pick a small angle $0<\alpha_{\min }<\pi / 2$, and let

$$
\mathcal{B}_{\alpha_{\text {min }}}\left(t_{j}\right)=\left\{q \in \mathcal{B}\left(t_{j}\right): \sin \left(\alpha_{q}\right) \geq \sin \left(\alpha_{\text {min }}\right)\right\} .
$$

(In other words, $\mathcal{B}_{\alpha_{\min }}(t)$ contains all points in $\mathcal{B}$ that are not in a conical region of half-width $\alpha_{\min }$, as shown in Fig. 4). For all $q \in \mathcal{B}\left(t_{j}\right), \cos \left(\alpha_{q}^{\prime}\right) \leq \cos \left(\alpha_{q}\right)$; moreover, for all $q \in \mathcal{B}_{\alpha_{\text {min }}}$

$$
\begin{aligned}
\cos \left(\alpha_{q}^{\prime}\right) & \leq \cos \left(\alpha_{q}\right)-\sin \left(\alpha_{\min }\right)\left(\alpha_{q}^{\prime}-\alpha_{q}\right) \\
& \leq \cos \left(\alpha_{q}\right)-\frac{\delta \sin \left(\alpha_{\min }\right)^{2}}{\|q-p\|} \\
& \leq \cos \left(\alpha_{q}\right)-\frac{\delta \sin \left(\alpha_{\min }\right)^{2}}{\operatorname{diam}(\mathcal{Q})+\delta} .
\end{aligned}
$$

Hence, summing over all $q \in \mathcal{B}\left(t_{j}\right)$, we get

$$
\sum_{q \in \mathcal{B}\left(t_{j}\right)} \cos \left(\alpha_{q}^{\prime}\right) \leq \sum_{q \in \mathcal{B}\left(t_{j}\right)} \cos \left(\alpha_{q}\right)-\sum_{q \in \mathcal{B}_{\alpha_{\min }}\left(t_{j}\right)} \frac{\delta \sin \left(\alpha_{\min }\right)^{2}}{\operatorname{diam}(\mathcal{Q})+\delta} .
$$

Observe now that in any case

$$
\left|\sum_{q \in \mathcal{B}\left(t_{j}\right)} \cos \left(\alpha_{q}\right)\right| \leq 1
$$

(it is zero in case $p^{*}\left(t_{j}\right) \notin \mathcal{B}\left(t_{j}\right)$, and bounded in absolute value by one if $\left.p^{*}\left(t_{j}\right) \in \mathcal{B}\left(t_{j}\right)\right)$. Therefore, rearranging (16)

$$
\begin{aligned}
& \operatorname{card}\left(\mathcal{B}_{\alpha_{\text {min }}}\left(t_{j}\right)\right) \frac{\delta \sin \left(\alpha_{\min }\right)^{2}}{\operatorname{diam}(\mathcal{Q})+\delta} \\
& \quad \leq \sum_{q \in \mathcal{B}\left(t_{j}\right)} \cos \left(\alpha_{q}\right)-\sum_{q \in \mathcal{B}\left(t_{j}\right)} \cos \left(\alpha_{q}^{\prime}\right) \leq 2 .
\end{aligned}
$$

Solving this inequality with respect to $\delta$ we get

$$
\delta \leq \frac{2 \operatorname{diam}(\mathcal{Q})}{\operatorname{card}\left(\mathcal{B}_{\alpha_{\min }}\left(t_{j}\right)\right) \sin \left(\alpha_{\min }\right)^{2}-2} .
$$

Since (i) $\alpha_{\min }$ is a positive constant, (ii) $p^{*}\left(t_{j}\right)$ is in the interior of $\mathcal{Q}$, and (iii) $\lim _{j \rightarrow \infty} \operatorname{card}\left(\mathcal{B}\left(t_{j}\right)\right)=+\infty$, the right hand side of (17) converges to zero with probability one. Since the bound holds for all points $p \in C\left(t_{j}\right)$, for all $j \in \mathbb{N}$, the claim follows.

In the previous proposition, we have proven that $\| p^{*}\left(t_{j+1}\right)-$ $p^{*}\left(t_{j}\right) \|$ tends to zero a.s. as $j \rightarrow \infty$, under some natural assumptions on the distribution $\varphi$ and its support. Unfortunately this is not sufficient to prove that the sequence $\left\{p^{*}\left(t_{j}\right)\right\}_{t_{j} \in \mathbb{N}}$ is Cauchy; convergence of the sequence is however ensured by the following.

Proposition 10: The sequence $\left\{p^{*}\left(t_{j}\right)\right\}_{j \in \mathbb{N}}$ converges almost surely.

Proof: Since the sequence $\left\{p^{*}\left(t_{j}\right)\right\}_{j \in \mathbb{N}}$ takes value in a compact set (the closure of $\operatorname{Supp}(\varphi)$ ), by the BolzanoWeirstrass theorem there exists a subsequence converging to a limit point $\hat{p}^{*}$ in the compact set. Construct from $\left\{p^{*}\left(t_{j}\right)\right\}_{j \in \mathbb{N}}$ a maximal subsequence converging to $\hat{p}^{*}$, and call $J$ the set of indices of this maximal subsequence. If the original sequence $\left\{p^{*}\left(t_{j}\right)\right\}_{j \in \mathbb{N}}$ is not converging to $\hat{p}^{*}$, then there exists an $L>0$ 
such that $\left\|p^{*}\left(t_{j}\right)-\hat{p}^{*}\right\| \geq L$, for any $j \in \mathbb{N} \backslash J$, and this set of indices is unbounded. Take $\epsilon:=L / 3>0$. We have that $\left\|p^{*}\left(t_{k-1}\right)-\hat{p}^{*}\right\| \leq \epsilon$ for any sufficiently large $(k-1) \in J$; moreover, $\left\|p^{*}\left(t_{k-1}\right)-p^{*}\left(t_{k}\right)\right\| \leq \epsilon$, a.s. by Proposition (9). Choose a sufficiently large $(k-1) \in J$, such that $k \in \mathbb{N}-J$ (this is always possible since the complementary set of $J$ is unbounded by the assumption of non-convergence). But now, we have

$$
\begin{gathered}
L \leq\left\|p^{*}\left(t_{k}\right)-\hat{p}^{*}\right\| \\
\leq\left\|p^{*}\left(t_{k}\right)-p^{*}\left(t_{k-1}\right)\right\|+\left\|p^{*}\left(t_{k-1}\right)-\hat{p}^{*}\right\| \leq \frac{2}{3} L
\end{gathered}
$$

which is a contradiction.

\section{Convergence to the Generalized Median}

By the discussion in the previous section, we know that the reference points of all agents that visit an unbounded number of targets converge to a well-defined limit. So do, trivially, the reference points of all agents that visit a bounded set of targets. Hence, we know that the sequence of reference points $p_{i}^{*}\left(t_{j}\right)$ converges to a limit $\hat{p}_{i}^{*}$, almost surely for all $i \in\{1, \ldots, m\}$. Let us denote by $\mathcal{V}_{i}\left(p^{*}\left(t_{j}\right)\right)$ the Voronoi region associated to the generator $p_{i}^{*}\left(t_{j}\right)$, and by $\mathcal{V}_{i}\left(\hat{p}^{*}\right)$ the Voronoi region corresponding to the limit point $\hat{p}_{i}^{*}$.

Proposition 11: If the limit reference points $\hat{p}^{*}=$ $\left(\hat{p}_{1}^{*}, \ldots, \hat{p}_{2}^{*}\right)$ are distinct, then the sequence of Voronoi partitions $\left\{\mathcal{V}\left(p^{*}\left(t_{j}\right)\right)\right\}_{j \in \mathbb{N}}$ converges to the Voronoi partition generated by the limit of reference points, i.e.

$$
\lim _{j \rightarrow \infty} \mathcal{V}_{i}\left(p^{*}\left(t_{j}\right)\right)=\mathcal{V}_{i}\left(\hat{p}^{*}\right), \quad \text { a.s. }
$$

Proof: The boundaries of regions in a Voronoi partition are algebraic curves that depend continuously on the generators, as long as these are distinct. Hence, under this assumption, almost sure convergence of the generators implies the almost sure convergence of the Voronoi regions.

As a next step, we wish to understand what is the relation between the asymptotic reference positions and their associated Voronoi regions. More precisely, let $\mathcal{A} \subset\{1, \ldots, m\}$ be the subset of indices of agents that visit an unbounded number of targets; we want to prove that $\hat{p}_{i}^{*}$ is indeed the generalized median $\bar{p}_{i}$ associated to agent $i$, with respect to the limiting set $\mathcal{V}_{j}\left(\hat{p}^{*}\right)$ and distribution $\varphi(x), \forall i \in \mathcal{A}$. First we need the following technical result.

Lemma 12: Let $\left\{f_{i}\right\}_{i \in \mathbb{N}}: \mathcal{Q} \rightarrow \mathbb{R}$ be a sequence of strictly convex continuous functions, defined on a common compact subset $\mathcal{Q} \subset \mathbb{R}^{n}$. Assume that each $f_{i}$ has a unique $x_{i}:=$ $\arg \min _{x} f_{i}$ belonging to the interior of $\Omega$ for any $i$ and that this sequence of function converges uniformly to a continuous strictly convex function $f$ admitting a unique minimum point $\bar{x}$ belonging also to the interior of $\Omega$. Then $\lim _{i} \rightarrow \infty x_{i}=\bar{x}$.

Proof: Since $\left\{f_{i}\right\}_{i \in \mathbb{N}}$ converges uniformly to $f$, then for any $\epsilon>0$, there exists an $I(\epsilon)$ such that for any $i \geq I(\epsilon)$, $\left\|f_{i}-f\right\| \leq \epsilon$ uniformly in $x \in \Omega$. Let $m:=f(\bar{x})$, the minimum value achieved by $f$. Consider the set

$$
U_{\epsilon}:=\{x \in \Omega \text { such that } f(x) \leq m+2 \epsilon\} .
$$

Since $f$ is strictly convex, for $\epsilon$ sufficiently small, $U_{\epsilon}$ will be a compact subset contained in $\Omega$. Moreover, since $f$ is strictly convex, we have that $U_{\epsilon}$ is strictly included in $U_{\epsilon^{\prime}}$, whenever $\epsilon^{\prime}>\epsilon$ and both are sufficiently small. It is also clear that $\lim _{\epsilon \rightarrow \infty} U_{\epsilon}=\bar{x}$ (nested strictly decreasing sequence of compact subsets all containing the point $\bar{x}$ ). If $\left\|f_{i}-f\right\| \leq \epsilon$, we claim that $x_{i} \in U_{\epsilon}$; we prove this by contradiction. Since $f_{i} \geq f-\epsilon$, if $x_{i}$ does not belong to $U_{\epsilon}$, then $\min \left(f_{i}\right)=f_{i}\left(x_{i}\right)>m+\epsilon$ (this is just because $U_{\epsilon}$ is simply the set where the function $(f-\epsilon) \leq m+\epsilon)$. But since $f_{i} \leq f+\epsilon$ it turns out that $f_{i}(\bar{x}) \leq m+\epsilon<\min \left(f_{i}\right)$, which is a contradiction.

We conclude this section with the following:

Proposition 13: Assume that all agents in $\mathcal{A}$ are able to return infinitely often to their reference point between visiting two targets. Then, the limit reference points of such agents coincide, almost surely, with the generalized medians of their limit Voronoi regions, i.e.

$$
\hat{p}_{i}^{*}=\arg \min _{p \in \Omega} \int_{\mathcal{V}_{i}\left(\hat{p}^{*}\right)}\|p-q\| \varphi(q) d q, \quad \text { a.s. }, \quad \forall i \in \mathcal{A} .
$$

Proof: For any $i \in \mathcal{A}$, define the functions $f_{i, t_{j}}(p):=$ $1 / j \sum_{q \in \mathcal{B}_{i}\left(t_{j}\right)}\|p-q\|$ and $f_{i}(p):=\int_{\mathcal{V}_{i}\left(\hat{p}^{*}\right)}\|p-q\| \varphi(q) d q$. These functions are continuous and well defined over $\Omega$. We restrict their domains of definition to the compact set $\mathcal{Q}=\operatorname{Supp}(\varphi)$. These functions are also strictly convex and have unique minima in the interior of $\mathcal{Q}$. Let us notice that, with our previous notations, we have that $p_{i}^{*}\left(t_{j}\right)=\arg \min f_{i, t_{j}}(p)$ and $\bar{p}_{i}=\arg \min f_{i}(p)$. Observe that the functions $f_{i, t_{j}}(p)$ and $f_{i}(p)$ can be considered random variables with respect to a probability space whose space of events coincide with all possible realizations of target sequences. Consider a restriction of these random variables to a new probability space whose space of events coincide with all possible realizations of target sequences, for which the corresponding FT points converge to a limiting point. On this new probability space the random variable $f_{i}(p)$ becomes a deterministic function which is the expected value of the random variables $f_{i, t_{j}}(p)$. Since $\mathcal{Q}$ is compact, it is immediate to see that $f_{i, t_{j}}(p)$ has finite expectation and variance over this reduced probability space, and by the Strong Law of Large Numbers we can conclude that almost surely (over this reduced probability space) $f_{i, t_{j}}(p)$ converge pointwise to $f_{i}(p)$. To show that $f_{i, t_{j}}(p)$ converges pointwise to $f_{i}(p)$ over the original probability space, it is sufficient to observe the following. The original probability space is the probability space whose space of events coincide with all possible realizations of target sequences. We already know that almost surely the FT points associated to any possible realization of target sequences will converge. So we can fiber the space of events of the first probability space into spaces of events of reduced probability spaces, except for a set of measure zero. This is sufficient to prove that $f_{i, t_{j}}(p)$ converge pointwise to $f_{i}(p)$ almost surely with respect to all possible realizations of target sequences.

Now that we have proved that almost surely the sequence $\left\{f_{i, t_{j}}(p)\right\}_{t_{j} \in \mathbb{R}}$ converges pointwise to $f_{i}(p)$, we prove that it does converge uniformly. To do this, we use a theorem from [28], which is usually devoted to Dini-Arzelà. The theorem 
states the following: an equicontinuous sequence of functions converges uniformly to a continuous function on a compact set $\mathcal{Q}$ if and only if it converges pointwise to a continuous function on the same compact set. Our sequence $f_{i, t_{j}}(p)$ is equicontinuous if $\forall \epsilon>0$ and $\forall p \in \mathcal{Q}$ there exists a $\delta>0$ such that for all $j \in\{1, \ldots, n\}$ and for all $p^{\prime} \in \mathcal{Q}$ with $\left\|p^{\prime}-p\right\| \leq \delta$, we have $\left\|f_{i, t_{j}}(p)-f_{i, t_{j}}\left(p^{\prime}\right)\right\| \leq \epsilon$; observe that $\delta$ is independent on $j$, while in general it will depend on $\epsilon$ and on $p$. Now we have

$$
\left\|f_{i, t_{j}}(p)-f_{i, t_{j}}\left(p^{\prime}\right)\right\| \leq \frac{1}{j} \sum_{q \in \mathcal{B}_{i}\left(t_{j}\right)}\|p-q\|-\left\|p^{\prime}-q\right\| .
$$

Using

$$
\|q-p\|=\left\|q-p^{\prime}+p^{\prime}-p\right\| \leq\left\|q-p^{\prime}\right\|+\left\|p-p^{\prime}\right\|
$$

it is immediate to see that

$$
\left\|f_{i, t_{j}}(p)-f_{i, t_{j}}\left(p^{\prime}\right)\right\| \leq \frac{1}{j} \sum_{q \in \mathcal{B}_{i}\left(t_{j}\right)}\left\|p-p^{\prime}\right\| \leq\left\|p-p^{\prime}\right\| .
$$

So it is sufficient to take $\delta=\epsilon$ in the previous definition and $\delta$ does not depend on $j$. So the sequence is equicontinuous and the pointwise convergence is upgraded to uniform convergence.

We already know that almost surely the points $p_{i}^{*}\left(t_{j}\right)$ do converge to points $\hat{p}_{i}^{*}$ (Proposition (10)); therefore we can claim that $\hat{p}_{i}^{*}=\bar{p}_{i}$, simply applying Lemma (12), which requires the uniform convergence. Thus we can claim that the reference position of each agent which services infinitely many targets following our algorithm converges to the generalized median of its Voronoi region, almost surely.

\section{Fairness}

In this section, we prove that, as long as $\varphi$ is strictly positive over a convex set, both policies introduced in Section III are fair.

Proposition 14 (Fairness): If $\mathcal{Q}=\operatorname{Supp}(\varphi)$ is convex, all agents eventually visit an unbounded number of targets, almost surely, i.e.

$$
\lim _{t \rightarrow+\infty} \operatorname{card}\left(\mathcal{B}_{i}(t)\right)=+\infty, \quad \text { a.s., } \forall i \in\{1, \ldots, m\} .
$$

Proof: Under either policy, each agent will pursue and is guaranteed to service the nearest target in its own Voronoi region. Hence, in order to show that an agent services an unbounded number of targets, it is sufficient to show that the probability that the next target be generated within its Voronoi region remains strictly positive, i.e., that

$$
\int_{\mathcal{V}_{i}(p(t))} \varphi(q) d q>0
$$

Since $\mathcal{Q}$ is convex, and all agents move towards the nearest target, at least initially, all agents will eventually enter $\mathcal{Q}$, and remain within it. Let us denote with $P_{i j}$ the probability that agent $i$ visits the $j$ th target. For any $i$ this probability is always strictly positive. Indeed, even if it happens that some agents are servicing simultaneously the same target (simply because the service request appears at the boundary of two different Voronoi regions), this does not mean that their reference points have to coincide. For the reference points of two agents eventually to coincide, it must happen that they are servicing infinitely many often and simultaneously the same target. Since the boundaries of Voronoi regions have measure zero and $\varphi$ is a continuous distribution without singular components, we can claim that $\lim _{j \rightarrow+\infty} P_{i j}>0$ almost surely. Now call $P=\min _{i=1, \ldots, m} \lim _{j \rightarrow+\infty} P_{i j}$. Then $P$ is strictly positive almost surely. Therefore, the probability that the $i$-th agent does not visit an unbounded number of targets is bounded from above by $\lim _{j} \rightarrow+\infty \prod_{k=1}^{j}(1-P)^{k}=0$, almost surely.

\section{E. Efficiency}

In this section, we will prove that the system time provided by both the algorithms in Section III converges to a critical point (either a saddle point or a local minimum) with high probability as $\lambda \rightarrow 0^{+}$.

In the preceding sections, we have proved that - as long as each agent is able to return to its reference point between servicing two targets, infinitely often-the reference points $p_{i}^{*}\left(t_{j}\right)$ converge to points $\hat{p}_{i}^{*}$, which generate a MVT. In such case, we know that the average time of service will converge to

$$
\begin{aligned}
\bar{T}_{\pi} & =\int_{\Omega} \min _{i=1, \ldots, m}\left\|\hat{p}_{i}^{*}-q\right\| \varphi(q) d q \\
& =\sum_{i=1}^{m} \int_{\mathcal{V}_{i}\left(\hat{p}^{*}\right)}\left\|\hat{p}_{i}^{*}-q\right\| \varphi(q) d q .
\end{aligned}
$$

Consider now functions $\mathcal{H}_{m}$ of the form

$$
\begin{aligned}
\mathcal{H}_{m}\left(p_{1}, \ldots, p_{m}\right) & =\int_{\Omega} \min _{i=1, \ldots, m}\left\|p_{i}-q\right\| \varphi(q) d q \\
& =\sum_{i=1}^{m} \int_{\mathcal{V}_{i}(p)}\left\|p_{i}-q\right\| \varphi(q) d q .
\end{aligned}
$$

Observe that $\bar{T}_{\pi}$ belongs to the class of functions of the form $\mathcal{H}_{m}$ where each point $p_{i}$ is constrained to be the generalized median of the corresponding Voronoi region (i.e., $\mathcal{V}(p)$ is a MVT).

We want to prove that $\bar{T}_{\pi}$ is a critical point of $\mathcal{H}_{m}$. To do so, we consider an extension of $\mathcal{H}_{m}$, i.e., a functional $\mathcal{K}_{m}$ defined as follows:

$\mathcal{K}_{m}\left(p_{1}, \ldots, p_{m}, \mathcal{V}_{1}, \ldots, \mathcal{V}_{m}\right):=\sum_{i=1}^{m} \int_{y \in \mathcal{V}_{i}}\left\|y-p_{i}\right\| \varphi(y) d y$.

Observe that in this case the regions $\left\{\mathcal{V}_{i}\right\}_{i=1, \ldots, m}$ are not restricted to form a MVT with respect to the generators $\left\{p_{i}\right\}_{i=1, \ldots, m}$. Thus we can view the functional $\mathcal{H}_{m}$ we are interested in as a constrained form of the unconstrained functional $\mathcal{K}_{m}$. It turns out therefore that critical points of $\mathcal{K}_{m}$ are also critical points of $\mathcal{H}_{m}$. With respect to critical points of $\mathcal{K}_{m}$ we have the following result:

Proposition 15: Let $\left\{p_{i}\right\}_{i=1, \ldots, m}$ denote any set of $m$ points belonging to $\operatorname{Supp}(\varphi)$ and let $\left\{\mathcal{V}_{i}\right\}_{i=1, \ldots, m}$ denote any tessellation of $\operatorname{Supp}(\varphi)$ into $m$ regions. Moreover, let us define $\mathcal{K}_{m}$ as above. Then a sufficient condition for $\left\{p_{1}, \ldots, p_{m}, \mathcal{V}_{1}, \ldots, \mathcal{V}_{m}\right\}$ to be a critical point (either a saddle point or a local minimum), is that the $\mathcal{V}_{i}$ 's are the Voronoi regions corresponding to the $p_{i}$ 's, and, simultaneously, the $p_{i}$ 's are the generalized median of the corresponding $\mathcal{V}_{i}$ 's. 
Proof: Consider first the variation of $\mathcal{K}_{m}$ with respect to a single point, say $p_{i}$. Now let $v$ be a vector in $\mathbb{R}^{2}$, such that $p_{i}+\epsilon v \in \Omega$. Then we have

$$
\begin{aligned}
\mathcal{K}_{m}\left(p_{i}+\epsilon v\right)- & \mathcal{K}_{m}\left(p_{i}\right) \\
& =\int_{y \in \mathcal{V}_{i}}\left\{\left\|y-p_{i}-\epsilon v\right\|-\left\|y-p_{i}\right\|\right\} \varphi(y) d y
\end{aligned}
$$

where we have not listed the other variables on which $\mathcal{K}_{m}$ depends since they remain constant in this variation. By the very form of this variation, it is clear that if the point $p_{i}$ is the generalized median for the fixed region $\mathcal{V}_{i}$, we will have that $\mathcal{K}_{m}\left(p_{i}+\epsilon v\right)-\mathcal{K}_{m}\left(p_{i}\right)>0$, for any $v$. Now consider the points $\left\{p_{i}\right\}_{i=1, \ldots, m}$ fixed and consider a tessellation $\left\{\mathcal{U}_{i}\right\}_{i=1, \ldots, m}$ different from the Voronoi regions $\left\{\mathcal{V}_{i}\right\}_{i=1, \ldots, m}$ generated by the points $p_{i}$ 's. We compare the value of $\mathcal{K}_{m}\left(p_{1}, \ldots, p_{m}, \mathcal{V}_{1}, \ldots, \mathcal{V}_{m}\right)$, with the value of $\mathcal{K}_{m}\left(p_{1}, \ldots, p_{m}, \mathcal{U}_{1}, \ldots, \mathcal{U}_{m}\right)$. Consider those $y$ which belong to the Voronoi region $\mathcal{V}_{j}$ generated by $p_{j}$, and possibly not to the Voronoi region of another $p_{i}$. Anyway, since $\mathcal{U}_{i}$ is not a Voronoi tessellation, it can happen that in any case these $y$ belong to $\mathcal{U}_{i}$. Thus for these particular $y$ 's we have $\varphi(y)\left\|y-p_{j}\right\| \leq \varphi(y)\left\|y-p_{i}\right\|$. Moreover, since $\left\{\mathcal{U}_{i}\right\}_{i=1, \ldots, m}$ are not the Voronoi tessellation associated to the $p_{i}$ 's, the last inequality must be strict over some set of positive measure. Thus we have that $\mathcal{K}_{m}\left(p_{1}, \ldots, p_{m}, \mathcal{V}_{1}, \ldots, \mathcal{V}_{m}\right)<$ $\mathcal{K}_{m}\left(p_{1}, \ldots, p_{m}, \mathcal{U}_{1}, \ldots, \mathcal{U}_{m}\right)$, and therefore $\mathcal{K}_{m}$ is minimized, keeping fixed the $p_{i}$ 's exactly when the subset $\mathcal{V}_{i}$ 's are chosen to be the Voronoi regions associated with the point $p_{i}$ 's.

By the previous proposition and by the fact that critical points of the unconstrained functional $\mathcal{K}_{m}$ are also critical points of the constrained functional $\mathcal{H}_{m}$, we have that the MVT are always critical points for the functional $\mathcal{H}_{m}$, and in particular $\bar{T}_{\pi}$ is either a saddle point or a local minimum for the functional $\mathcal{H}_{m}$.

We can now conclude with following:

Theorem 16 (Efficiency): The system time provided by the no-communication policy $\pi_{\mathrm{nc}}$ and by the sensor-based policy $\pi_{\mathrm{sb}}$ converges to a critical point (either a saddle point or a local minimum) with high probability as $\lambda \rightarrow 0^{+}$.

Proof: Combining results in Propositions 10 and 13 we conclude that the reference points of all agents that visit an unbounded number of targets converge to a MVT, almost surely-provided agents can return to the reference point between visiting targets. Moreover, the fairness result in Proposition 14 shows that in fact all agents do visit an unbounded number of targets almost surely; as a consequence of Proposition 15 the limit configuration is indeed a critical point for the system time. Since agents return infinitely often to their reference positions with high probability as $\lambda \rightarrow 0$, the claim is proven.

Thus we have proved that the suggested algorithm enables the agents to realize a coordinated task, such that "minimizing" the cost function without explicit communication, or with mutual position knowledge only. Let us underline that, in general, the achieved critical point strictly depends on the initial positions of the agents inside the environment $\Omega$. It is known that the function $\mathcal{H}_{m}$ admits (not unique, in general) global minima, but the problem to find them is NP-hard.
Remark 17: We can not exclude that the algorithm so designed will converge indeed to a saddle point instead of a local minimum. This is due to the fact that the algorithm provides a sort of implementation of the steepest descent method, where, unfortunately we are not following the steepest direction of the gradient of the function $\mathcal{H}_{m}$, but just the gradient with respect to one of the variables.

On the other hand, since the algorithm is based on a sequence of targets and at each phase we are trying to minimize a different cost function, it can be proved that the critical points reached by this algorithm are no worse than the critical points reached knowing a priori the distribution $\varphi$. This is a remarkable result proved in a different context in [29], where an example is presented in which the use of a sample sequence provides a better result (with probability one) than the a priori knowledge of $\varphi$. In that specific example the algorithm with the sample sequence does converge to a global minimum, while the algorithm based on the a priori knowledge of the distribution $\varphi$ gets stuck in a saddle point.

\section{F. A Comparison With Algorithms for Vector Quantization and Centroidal Voronoi Tessellations}

The use of Voronoi tessellations is ubiquitous in many fields of science. In the available literature, most of the analysis is devoted to applications of centroidal Voronoi tessellations, i.e., Voronoi tessellations such that

$$
p_{i}=\arg \min _{s \in \mathbb{R}^{n}} \int_{\mathcal{V}_{i}\left(p_{1}, p_{2}, \ldots, p_{m}\right)}\|s-q\|^{2} \varphi(q) d q
$$

for each $i \in\{1, \ldots, m\}$. A popular algorithm due to Lloyd [20] is based on the iterative computation of centroidal Voronoi tessellations. The algorithm can be summarized as follows. Pick $m$ generator points, and consider a large number $n$ of samples from a certain distribution. At each step of the algorithm generators are moved towards the centroid of the samples inside their respective Voronoi region. The algorithm terminates when each generator is within a given tolerance from the centroid of samples in its region, thus obtaining a centroidal Voronoi tessellation weighted by the sample distribution. An application to coverage problems in robotics and sensor networks of Lloyd's algorithm is available in [14].

The algorithms we introduced in this paper are more closely related to an algorithm due to MacQueen [21], originally designed as a simple online adaptive algorithm to solve clustering problems, and later used as the method of choice in several vector quantization problems where little information about the underlying geometric structure is available. MacQueen's algorithm can be summarized as follows. Pick $m$ generator points. Then iteratively sample points according to the probability density function $\varphi$. Assign the sampled point to the nearest generator, and update the latter by moving it in the direction of the sample. In other words, let $q_{j}$ be the $j$-th sample, let $i^{*}(j)$ be the index of the nearest generator, and let $c=\left(c_{1}, \ldots, c_{m}\right)$ be a counter vector, initialized to a vector of ones. The update rule takes the form

$$
p_{i^{*}(j)} \leftarrow \frac{q_{j}+c_{i^{*}(j)} p_{i^{*}(j)}}{c_{i^{*}(j)}+1}, \quad c_{i^{*}(j)} \leftarrow c_{i^{*}(j)}+1 .
$$


The process is iterated until some termination criterion is reached. Compared to Lloyd's algorithm, MacQueen's algorithm has the advantage to be a learning adaptive algorithm, not requiring the a priori knowledge of the distribution of the objects.

The algorithm we propose is very similar in spirit to MacQueen's algorithm, however, there is a remarkable difference. MacQueen's algorithm deals with centroidal Voronoi tessellations, thus with the computation of $k$-means. Our algorithm instead is based on MVT, and on the computation of $k$-medians. In general, very little is known for Voronoi diagrams generated using simply the Euclidean distance instead of the square of the Euclidean distance. For instance, the medians of a sequence of points can exhibit a quite peculiar behavior if compared to the one of the means. Consider the following example. Given a sequence of points $\left\{q_{i}\right\}_{i \in \mathbb{N}}$ in a compact set $K \subset \mathbb{R}^{2}$, we can construct the induced sequence of means

$$
m_{N}:=\frac{1}{N} \sum_{i=1}^{N} q_{j}
$$

and analogously the induce sequence of FT points we considered in the previous sections. Call $F T_{N}$ the FT point corresponding to the first $N$ points of the sequence $\left\{q_{i}\right\}_{i \in \mathbb{N}}$. We want to point out that induced sequence $\left\{m_{j}\right\}$ and $\left\{F T_{j}\right\}$ have a very different behaviour. Indeed, the induced sequence of means will always converge as long as the points $q_{j}$ 's belong to a compact set. To see this, just observe that if $\operatorname{diam}(K) \leq L$, then $\left\|m_{j}\right\| \leq L$. Moreover, it is immediate to see that $\| m_{N+1}-$ $m_{N} \| \leq 2 L / N$. Then one can conclude using the same argument of Proposition 10. On the other hand, one can construct a special sequence of points $q_{j}$ 's in a compact set $K$ for which the induced sequence of FT points does not converge. Therefore, it should be clear that the use of MVT instead of centroidal Voronoi tessellations makes much more difficult to deal with the technical aspects of the algorithm such as its convergence.

\section{A GAME-Theoretic Point of VieW}

In previous sections, we proposed two policies, $\pi_{\mathrm{sb}}$ and $\pi_{\mathrm{nc}}$, which yield the $\hat{p}^{*}$ reference points under light load. A key component of the two policies was the update rule for the reference points. In this section we provide a game-theoretic interpretation of the $\hat{p}^{*}$ reference points. In particular, we frame our presentation along the works [8], [30], in which game-theoretic point of view has been introduced in the study of cooperative control and strategic coordination of decentralized networks of multi-agents systems. We prove that the $\hat{p}^{*}$ reference points, under light load conditions, are an efficient pure Nash equilibria in a multi-player game setting where each agent is interested in maximizing its own utility. It turns out that, by just trying to maximize their own utility function, the agents will indeed maximize a common global utility function. The update rule for the reference point proposed as part of the two policies in the earlier sections can then be regarded as a mechanism for the vehicles to learn the $\hat{p}^{*}$ strategy.

We first formulate the game in the strategic form [31] as follows. Consider a scenario with the same workspace $\Omega$, density function $\varphi$ with support $\mathcal{Q}$ and the same stochastic process for generating service requests as described in the previous sections. We replace the terms service requests and targets with resources to fit better in the context of this section. The players of the game are the $m$ vehicles. The vehicles are assumed to be rational autonomous decision makers trying to maximize their own utility function. The resources offer rewards in a continuous fashion and the vehicles can collect these rewards by traveling to the resource locations. Every resource offers reward at a rate, which depends on the number of vehicles present at its location: the reward rate is unity when there is one vehicle and it is zero when there are more than one vehicles. Moreover, the life of the resource ends as soon as more than one vehicles are present at its location. The sensing and communication capabilities of the vehicles are as follows: i) the location of a resource is broadcast to all the vehicles as soon as it is generated; similarly, every vehicle is notified as soon as a resource ceases to exist, and ii) there is no explicit communication between the vehicles, i.e., the vehicles do not have knowledge about each other's position and no vehicle knows the identities of other vehicles visiting any resource location.

This setup can be understood to be an extreme form of congestion game [32], where the resource cannot be shared between agents and that the resource is cut off at the first attempt to share it. The total reward for vehicle $i$ from a particular resource is the time difference between its arrival and the arrival of the next vehicle, if $i$ is the first vehicle to reach the location of the resource, and zero otherwise. (Note that, since a vehicle cannot communicate with any other vehicle, it cannot determine if it will be the first one to reach the resource location when the location is broadcast to it).

We focus our analysis on the light load case here, i.e., when $\lambda \rightarrow 0^{+}$. Moreover, we assume that all the vehicles are present in $\mathcal{Q}$ at time $t=0$ and that there are no resources at time $t=0$. Hence, there will be utmost one active resource at any time almost surely. Note that these assumptions on the initial conditions are without any loss of generality in light of the discussion in the earlier sections. Since our focus is on the light load scenario, we let the strategy space of agent $i$ to be $\mathcal{Q}$ for all $i \in\{1, \ldots, m\}$. Specifically, the strategy of an agent $i$ denoted as $\pi_{i}$ is identified with a reference point in $\mathcal{Q}$ which the agent approaches in the absence of outstanding service requests. On the generation of a resource, the agents move directly towards its location and return back to the reference location once the resource ceases to be active. We will use $\pi_{-i} \in \mathcal{Q}^{m-1}$ to denote the strategy specification of all the agents, except agent $i$, i.e., $\pi_{-i}:=\left(\pi_{1}, \ldots, \pi_{i-1}, \pi_{i+1}, \ldots, \pi_{m}\right)$. Hence, we may write strategy vector $\pi$ as $\left(\pi_{i}, \pi_{-i}\right)$. Let $\mathcal{U}_{i}: \mathcal{Q}^{m} \rightarrow \mathbb{R}$ be the utility function of vehicle $i$. For a given strategy vector $\pi$, let $r_{i}(q, \pi)$ be the reward collected by agent $i$ for resource generated at location $q \in \mathcal{Q}$. In light of the discussion above, the utility function of an agent $i$ is defined as

$$
\mathcal{U}_{i}\left(\pi_{i}, \pi_{-i}\right)=\mathrm{E}_{q}\left[r_{i}(q, \pi)\right]
$$

Equation (20) implies that the goal of every vehicle is to maximize the expected value of the reward from the next resource. With this, we complete the formal description of the game at 
hand. For brevity in notation, we use $\mathcal{G}$ to denote this game. We now derive a working expression for the utility function.

As mentioned before, the reward for agent $i$ is the time till the arrival of the second agent at point $q$ if agent $i$ is the first to reach $q$ and zero otherwise. Since the vehicles move at unit speed, the reward for an agent $i$ can be written as $r_{i}(q, \pi)=$ $\max \left\{0, \min _{j \neq i}\left\|\pi_{j}-q\right\|-\left\|\pi_{i}-q\right\|\right\}$. The utility function for agent $i$ can then be written as

$$
\begin{aligned}
& \mathcal{U}_{i}\left(\pi_{i}, \pi_{-i}\right)=\mathrm{E}_{q}\left[\max \left\{0, \min _{j \neq i}\left\|\pi_{j}-q\right\|-\left\|\pi_{i}-q\right\|\right\}\right] \\
= & \int_{\mathcal{Q}} \max \left\{0, \min _{j \neq i}\left\|\pi_{j}-q\right\|-\left\|\pi_{i}-q\right\|\right\} \varphi(q) d q .
\end{aligned}
$$

However, we know that

$$
\begin{aligned}
& \max \left\{0, \min _{j \neq i}\left\|\pi_{j}-q\right\|-\left\|\pi_{i}-q\right\|\right\} \\
&= \begin{cases}\min _{j \neq i}\left\|\pi_{j}-q\right\|-\left\|\pi_{i}-q\right\|, & \text { if } q \in \mathcal{V}_{i}(\pi) \\
0, & \text { otherwise. }\end{cases}
\end{aligned}
$$

Substituting this into (21), we derive the following expression for the utility function

$$
\mathcal{U}_{i}\left(\pi_{i}, \pi_{-i}\right)=\int_{\mathcal{V}_{i}(\pi)}\left(\min _{j \neq i}\left\|\pi_{j}-q\right\|-\left\|\pi_{i}-q\right\|\right) \varphi(q) d q .
$$

We now prove that $\mathcal{G}$ belongs to a class of multi-player games called potential games. In a potential game, the difference in the value of the utility function for any agent for two different strategies, when the strategies of the other agents are kept fixed, is equal to the difference in the values of a potential function that depends only on the strategy vector and not on the label of any agent. The formal definition [33] is as follows.

Definition 18: A finite $m$-player game with strategy spaces $\left\{\Pi_{i}\right\}_{i=1}^{m}$ and utility functions $\left\{\mathcal{U}_{i}\right\}_{i=1}^{m}$ is a potential game if, for some potential function $\psi: \times_{i \in\{1, \ldots, m\}} \Pi_{i} \rightarrow \mathbb{R}$

$$
\mathcal{U}_{i}\left(\pi_{i}^{\prime}, \pi_{-i}\right)-\mathcal{U}_{i}\left(\pi_{i}^{\prime \prime}, \pi_{-i}\right)=\psi\left(\pi_{i}^{\prime}, \pi_{-i}\right)-\psi\left(\pi_{i}^{\prime \prime}, \pi_{-i}\right)
$$

for every player $i \in\{1, \ldots, m\}$, for every $\pi_{-i} \in \times_{j \neq i} \Pi$ and for every $\pi_{i}^{\prime}, \pi_{i}^{\prime \prime} \in \Pi_{i}$.

Proposition 19: $\mathcal{G}$ is a potential game.

Proof: The expression for the utility function of agent $i$, as given by (22), can be rewritten as

$$
\begin{aligned}
& \int_{\mathcal{V}_{i}(\pi)} \min _{j \neq i}\left\|\pi_{j}-q\right\| \varphi(q) d q-\int_{\mathcal{V}_{i}(\pi)}\left\|\pi_{i}-q\right\| \varphi(q) d q \\
& +\sum_{\substack{j=1 \\
j \neq i}}^{m} \int_{\mathcal{V}_{j}(\pi)}\left\|\pi_{j}-q\right\| \varphi(q) d q-\sum_{\substack{j=1 \\
j \neq i}}^{m} \int_{\mathcal{V}_{j}(\pi)}\left\|\pi_{j}-q\right\| \varphi(q) d q \\
& =\int_{\mathcal{V}_{i}(\pi)} \min _{j \neq i}\left\|\pi_{j}-q\right\| \varphi(q) d q+\sum_{\substack{j=1 \\
j \neq i}}^{m} \int_{\mathcal{V}_{j}(\pi)}\left\|\pi_{j}-q\right\| \varphi(q) d q \\
& -\sum_{j=1}^{m} \int_{\mathcal{V}_{j}(\pi)}\left\|\pi_{j}-q\right\| \varphi(q) d q=
\end{aligned}
$$

$$
\begin{aligned}
= & \int_{\mathcal{V}_{i}(\pi)} \min _{j \neq i}\left\|\pi_{j}-q\right\| \varphi(q) d q+\sum_{\substack{j=1 \\
j \neq i}}^{m} \int_{\mathcal{V}_{j}(\pi)} \min _{k \neq i}\left\|\pi_{k}-q\right\| \varphi(q) d q \\
& -\sum_{j=1}^{m} \int_{\mathcal{V}_{j}(\pi)}\left\|\pi_{j}-q\right\| \varphi(q) d q \\
= & \int_{\mathcal{Q}} \min _{j \neq i}\left\|\pi_{j}-q\right\| \varphi(q) d q-\sum_{j=1}^{m} \int_{\mathcal{V}_{j}(\pi)}\left\|\pi_{j}-q\right\| \varphi(q) d q .
\end{aligned}
$$

In the integrand of the first term in (23), $\min _{j \neq i}\left\|\pi_{j}-q\right\|$ is the distance from point $q$ to the closest among all the agents, except the $i$ th agent. We then consider the Voronoi partition with generators $\pi_{-i}=\pi \backslash \pi_{i}$, and let $\mathcal{V}_{j}\left(\pi_{-i}\right)$ be the corresponding Voronoi cell belonging to agent $j$. Equation (23) can then be written as

$\mathcal{U}_{i}\left(\pi_{i}, \pi_{-i}\right)=$

$\sum_{\substack{j=1 \\ j \neq i}}^{m} \int_{\mathcal{V}_{j}\left(\pi_{-i}\right)}\left\|\pi_{j}-q\right\| \varphi(q) d q-\sum_{j=1}^{m} \int_{\mathcal{V}_{j}(\pi)}\left\|\pi_{j}-q\right\| \varphi(q) d q$.

Note that the first term on the right hand side of (24) is independent of $\pi_{i}$. With this observation, consider the following potential function:

$$
\psi(\pi)=-\sum_{i=1}^{m} \int_{\mathcal{V}_{i}(\pi)}\left\|\pi_{i}-q\right\| \varphi(q) d q .
$$

The proposition then follows by combining (24) and (25) with the definition of a potential game.

It turns out that an agent's motive to maximize its own utility function is aligned with the global objective of minimizing the average system time. To formally establish this fact, we start with a couple of definitions. First, we extend the concept of a potential game.

Definition 20: A finite $m$-player game with strategy spaces $\left\{\Pi_{i}\right\}_{i=1}^{m}$ and utility functions $\left\{\mathcal{U}_{i}\right\}_{i=1}^{m}$ is an ordinal potential game if, for some potential function $\psi: \times_{i \in\{1, \ldots, m\}} \Pi_{i} \rightarrow \mathbb{R}$

$$
\mathcal{U}_{i}\left(\pi_{i}^{\prime}, \pi_{-i}\right)-\mathcal{U}_{i}\left(\pi_{i}^{\prime \prime}, \pi_{-i}\right)>0
$$

if and only if

$$
\psi\left(\pi_{i}^{\prime}, \pi_{-i}\right)-\psi\left(\pi_{i}^{\prime \prime}, \pi_{-i}\right)>0
$$

for every player $i \in\{1, \ldots, m\}$, for every $\pi_{-i} \in \times_{j \neq i} \Pi$ and for every $\pi_{i}^{\prime}, \pi_{i}^{\prime \prime} \in \Pi_{i}$.

Remark 21: Note that every potential game is also an ordinal potential game.

Definition 22: The set of agents utilities $\left\{\mathcal{U}_{i}\right\}_{i=1, \ldots, m}$ is aligned with the global utility $\mathcal{U}_{g}$ if and only if the game with utility functions $\left\{\mathcal{U}_{i}\right\}_{i=1, \ldots, m}$ is an ordinal potential game with $\mathcal{U}_{g}$ as a potential function.

For the game $\mathcal{G}$, define the global utility function to be the negative of the average system time under policy $\pi$, i.e., $\mathcal{U}_{g}(\pi) \equiv-\bar{T}_{\pi}$, which can be rewritten as

$$
\mathcal{U}_{g}(\pi)=-\sum_{i=1}^{m} \int_{\mathcal{V}_{i}(\pi)}\left\|\pi_{i}-q\right\| \varphi(q) d q .
$$


Proposition 23: The utility functions of the agents in $\mathcal{G}$ are aligned with its global utility function.

Proof: Comparing (26) with the expression of the potential function in (25), we observe that $\mathcal{U}_{g}(\pi)=\psi(\pi)$. Proposition 19 implies that $\mathcal{G}$ is a potential game, and hence an ordinal potential game, with $\psi$ as the potential function. The proposition then follows from Definition 22.

We now show that our utility function belongs to a class of utility functions called Wonderful Life Utility Functions [34].

Definition 24: Given a global utility function $\mathcal{U}_{g}\left(\pi_{i}, \pi_{-i}\right)$, the Wonderful Life (local) utility function is given by

$$
\mathcal{U}_{i}\left(\pi_{i} . \pi_{-i}\right)=\mathcal{U}_{g}\left(\pi_{i}, \pi_{-i}\right)-\mathcal{U}_{g}\left(\pi_{-i}\right)
$$

where $\mathcal{U}_{g}\left(\pi_{-i}\right)$ is the global utility in absence of agent $i$.

Remark 25: The Wonderful Life utility function measures the marginal contribution of an agent towards the global utility.

Proposition 26: The local utility function, as defined in (24) is a Wonderful Life utility function with respect to the global utility function defined in (26).

Proof: We refer to (24) in the proof of Proposition 19, where we derived an alternate expression for the local utility function. Comparing the two terms individually with the expression for the global utility function in (26), we get that

$$
\mathcal{U}_{i}\left(\pi_{i}, \pi_{-i}\right)=\mathcal{U}_{g}\left(\pi_{i}, \pi_{-i}\right)-\mathcal{U}_{g}\left(\pi_{-i}\right) .
$$

The proposition then follows from the definition of the Wonderful Life utility function.

The $\pi_{\mathrm{nc}}$ policy yields the equilibrium strategy $\hat{p}^{*}=$ $\left\{\hat{p}_{1}^{*}, \ldots, \hat{p}_{m}^{*}\right\}$ such that, for all $i \in\{1, \ldots, m\}, \hat{p}_{i}^{*}$ is the median of the Voronoi region $\mathcal{V}_{i}\left(\hat{p}^{*}\right)$. We now state and prove results regarding the efficiency and equilibrium status of the $\hat{p}^{*}$ strategy in the game theoretic setting of this section. We first state the following definition adapted from [8]:

Definition 27: A strategy $\tilde{\pi}$ is called a pure Nash equilibrium if, for all $i=\{1, \ldots, m\}$

$$
\mathcal{U}_{i}\left(\tilde{\pi}_{i}, \tilde{\pi}_{-i}\right)=\max _{\pi_{i} \in \Pi_{i}} \mathcal{U}_{i}\left(\pi_{i}, \tilde{\pi}_{-i}\right) .
$$

Moreover, a strategy $\pi$ is called efficient if there is no other strategy that yields higher utilities to all the agents.

Proposition 28: The $\hat{p}^{*}$ strategy is an efficient pure Nash equilibrium for the game $\mathcal{G}$.

Proof: For any $\pi_{-i}=\left\{p_{-i}, t_{-i}\right\}$

$$
\hat{p}_{i}^{*}=\operatorname{argmin}_{p_{i} \in \mathcal{Q}} \int_{\mathcal{V}_{i}(p)}\left\|p_{i}-q\right\| \varphi(q) d q .
$$

Combining this with the definition of an efficient Nash equilibrium, one arrives at the proposition.

Remark 29: Note that we do not claim in Proposition 28 that the $\pi^{*}$ strategy provides the unique efficient pure Nash equilibrium for the game $\mathcal{G}$. For instance to find other efficient pure Nash equilibria it is sufficient to modify the algorithm during the initial phases, when the FT points are not uniquely determined. These modifications produce different policy vectors which are anyway all efficient pure Nash equilibria, as it is immediate to see.
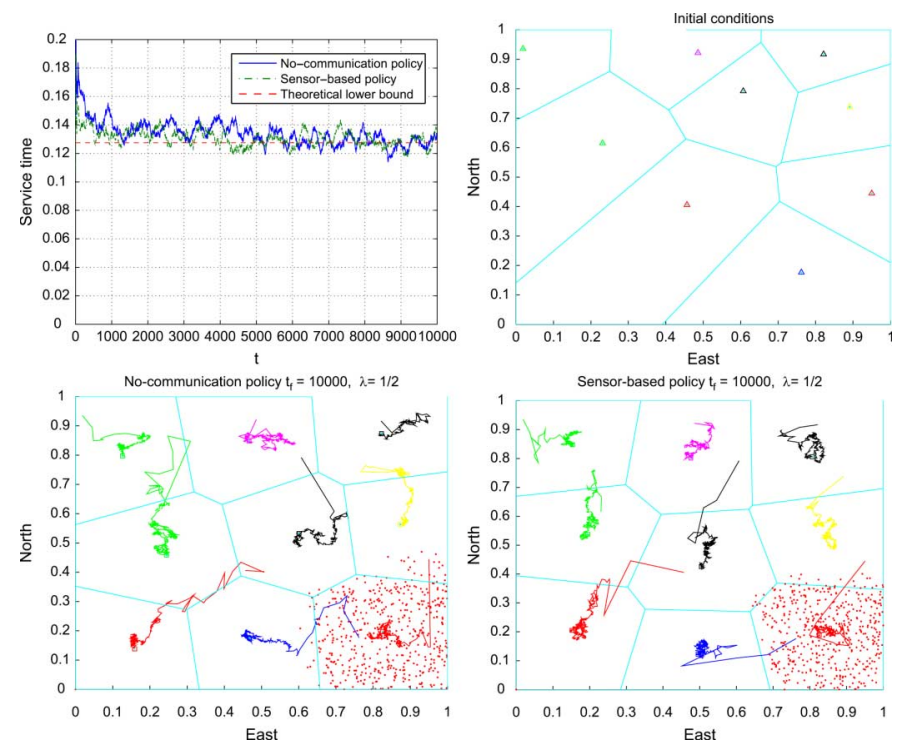

Fig. 5. Numerical simulation in the light-load case, for a uniform spatial distribution. Top left: The actual service times as a function of time, for the two policies, compared with the optimal system time. Top right: The initial configuration of the nine agents. Bottom left and right: Paths followed by the reference points up to $t=10^{4}$ (corresponding to approximately 5,000 targets), using the two policies. The locations of all targets visited by one of the agents are also shown.

In general, it is true that alignment does not prevent pure Nash equilibria from being suboptimal from the point of view of the global utility. Moreover, even efficient pure Nash equilibria (i.e., pure Nash equilibria which yield the highest utility to all agents) can be suboptimal from the perspective of the global utility function. Such a phenomenon is indeed what happens in our construction.

In the earlier sections, we proved that the update rule for the reference point which was part of both the policies converges to $\hat{p}^{*}$ almost surely under light load conditions. That update rule can then be thought of a learning algorithm for the agents to arrive at the efficient Nash equilibrium $\hat{p}^{*}$, even without explicit knowledge of the history of policies played by the other agents at every iteration.

\section{NUMERICAL RESUltS}

\section{A. Uniform Distribution, Light Load}

In the numerical experiments, we first consider $m=9$, choose $\mathcal{Q}$ as a unit square, and set $\varphi=1$ (i.e., we consider a spatially uniform target-generation process). This choice allows us to determine easily the optimal placement of reference points, at the centers of a tessellation of $\mathcal{Q}$ into nine equal squares, and compute analytically the optimal system time. In fact, it is known that the expected distance of a point $q$ randomly sampled from a uniform distribution within a square of side $L$ from the center of the square $c$ is

$$
\mathrm{E}[\|q-c\|]=\frac{\sqrt{2}+\log (1+\sqrt{2})}{6} L \approx 0.3826 L .
$$

The results for a small value of $\lambda$, i.e., $\lambda=0.5$, are presented in Fig. 5. The average service time converges to a value that is very close to the theoretical limit computed above, taking 

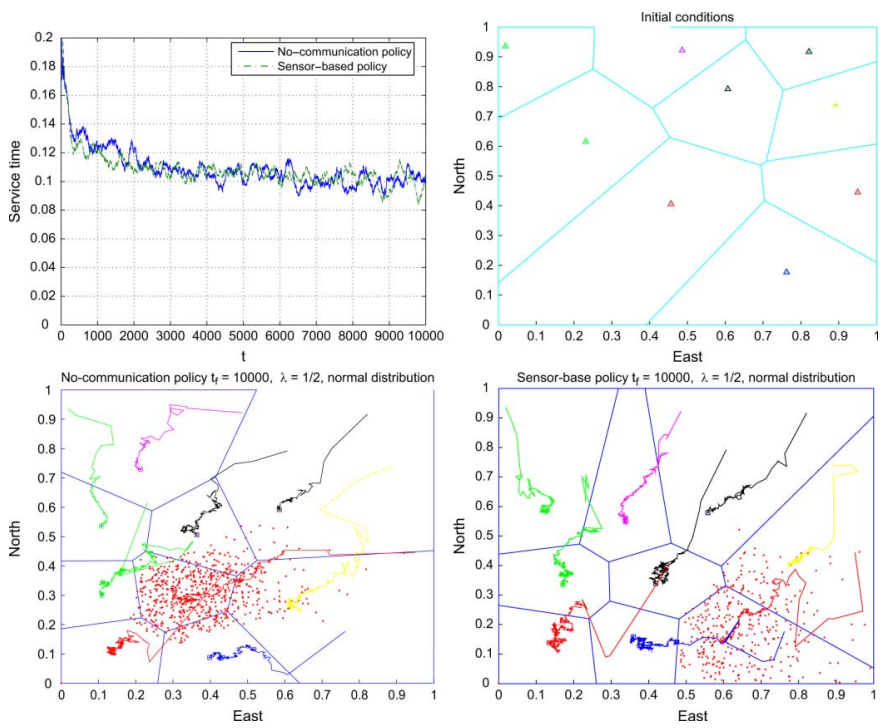

Fig. 6. Numerical simulation in the light-load case, for a normal spatial distribution. Top left: The actual service times as a function of time, for the two policies. Top right: The initial configuration of the nine agents. Bottom left and right: Paths followed by the reference points up to $t=10^{4}$ (corresponding to approximately 5,000 targets), using the two policies. The locations of all targets visited by one of the agents are also shown.

$L=1 / \sqrt{m}=1 / 3$. In both cases, the reference points converge-albeit very slowly - to the generators of a MVT, while the average system time quickly approaches the optimal value.

\section{B. Non-Uniform Distribution, Light Load}

We also present in Fig. 6 results of similar numerical experiments with a non-uniform distribution, namely an isotropic normal distribution centered at $(0.25,0.25)$, with standard deviation equal to 0.25 .

\section{Uniform Distribution, Dependency on the Target Generation Rate}

An interesting set of numerical experiments evaluates the performance of the proposed policies over a large range of values of the target generation rate $\lambda$. In Section IV, we proved the convergence of the system's behavior to an efficient steady state, with high probability as $\lambda \rightarrow 0^{+}$, as confirmed by the simulations discussed above. For large values of $\lambda$ however, the assumption that vehicles are able to return to their reference point breaks down, and the convergence result is no longer valid. In Fig. 7 we report results from numerical experiments on scenarios involving $m=3$ agents, and values of $\lambda$ ranging from $1 / 2$ to 32. In the figure, we also report the known (asymptotic) lower bounds on the system time (with 3 agents), as derived in [11], and the system time obtained with the proposed policies in a single-agent scenario.

The performance of both proposed policies is close to optimal for small $\lambda$, as expected. The sensor-based policy behaves well over a large range of target generation rates; in fact, the numerical results suggest that the policy provides a system time that is a constant-factor approximation of the optimum, by a factor of approximately 1.6 .

However, as $\lambda$ increases, the performance of the no-communication policy degrades significantly, almost approaching

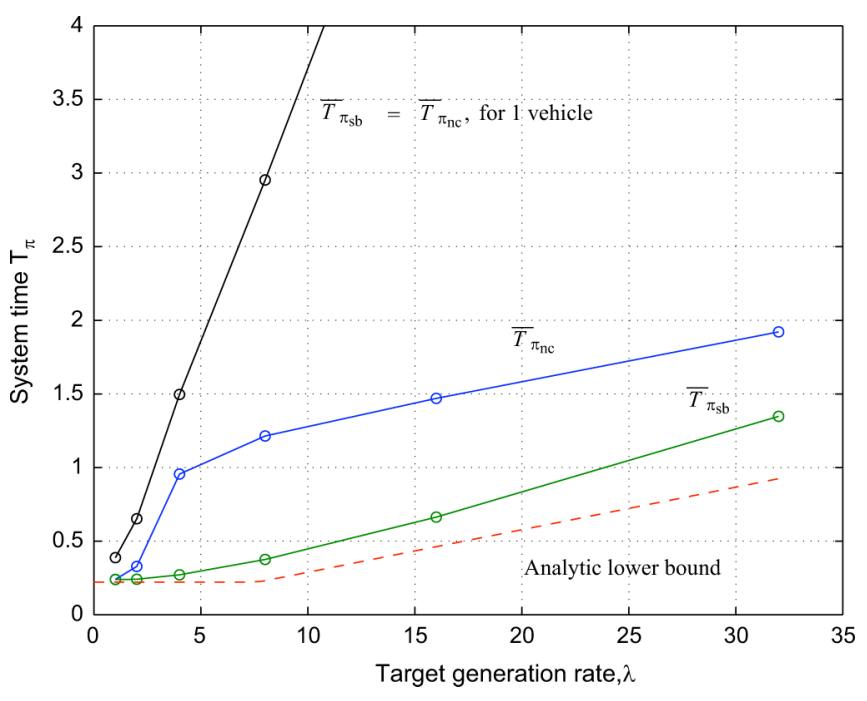

Fig. 7. System time provided by the policies proposed in this paper, as a function of the target generation rate $\lambda$. The system is composed of three vehicles, and the target points are generated uniformly in the unit square. Each data point is obtained taking the mean of the results obtained running one hundred times the system dynamics, for each specified policy and for each specified target generation rate $\lambda$. Therefore each data point is a Monte Carlo approximation of the theoretical system time corresponding to that policy and to that target generation rate.

the performance of a single-vehicle system over an intermediate range of values of $\lambda$. Our intuition in this phenomenon is the following. As agents do not return to their own reference points between visiting successive targets, their efficiency decreases since they are no longer able to effectively separate regions of responsibility. In practice-unless they communicate and concentrate on their Voronoi region, as in the sensor-based policy-agents are likely to duplicate efforts as they pursue the same target, and effectively behave as a single-vehicle system. Interestingly, this efficiency loss seems to decrease for large $\lambda$, and the numerical results suggest that the no-communication policy recovers a similar performance as the sensor-based policy in the heavy load limit.

Finally, let us add two remarks. The first concerns what happens if the agents are slower. After a suitable rescaling, reducing the agent velocities is equivalent to increase the target generation rate $\lambda$. As it is evident from Fig. 7 even when $\lambda$ increases both policies are stabilizing, in the sense that the system time is finite in both cases. On the other hand, the system time is much smaller for the sensor based policy when $\lambda>2.5$, or equivalently when the agents velocities are decreased. Therefore the sensor based policy performs better for bigger values of $\lambda$, that is to say for smaller agent velocities.

The second remark deals with the following question: what happens if $D(t)$ in not known, but only a delayed version of it is known to the agents? Assume that the agents know $D(t-$ $t_{d}$ ), where $t_{d}$ is a positive integrable random variable, that is assume that the delay with which the information about the set of outstanding targets reaches the agents, is a positive integrable random variable $t_{d}$. Assume moreover that $E\left[t_{d}\right]=\Delta$. It is immediate to see that the presence of this random delay will affect the performance of the system, namely the time of service, increasing it by an amount equal to $\Delta$. So for instance, the plots 
in Fig. 7 will shift upward of a quantity equal to $\Delta$, whenever the delay is modeled in this way.

\section{CONCLUSION}

In this paper, we considered two very simple strategies for multiple vehicle routing in the presence of dynamically-generated targets, and analyzed their performance in light load conditions, i.e., when the target generation rate is very small. The strategies we addressed in this paper are based on minimal assumptions on the ability of the agents to exchange information: in one case they do not explicitly communicate at all, and in the other case, agents are only aware of other agents' current location. A possibly unexpected and striking results of our analysis is the following: the collective performance of the agents using such minimal or no-communication strategies is (locally) optimal, and is in fact as good as that achieved by the best known decentralized strategies, see for instance [12] for a comparison. The proposed no-communication algorithm does converge to a (local) optimum, even though quite slowly compared to other policies. On the other hand, the proposed strategies do not rely on the knowledge of the target generation process, and make minimal assumptions on the target spatial distribution; in fact, the convexity and boundedness assumptions on the support of the spatial distribution can be relaxed, as long as path connectivity of the support, and absolute continuity of the distribution are ensured. Also, the distribution need not be constant: Indeed, the algorithm will provide a good approximation to a local optimum for the cost function as long as the characteristic time it takes for the target generation process to vary significantly is much greater than the relaxation time of the algorithm.

The proposed strategies are very simple to implement, as they only require storage of the coordinates of points visited in the past and simple algebraic calculations; the "sensor-based" strategy also require a device to estimate the position of other agents. Simple implementation and the absence of active communication makes the proposed strategies attractive, for example, in embedded systems and stealthy applications. The game-theoretic interpretation of our results also provides some insight into how territorial, globally optimal behavior can arise in a population of selfish but rational individuals even without explicit mechanisms for territory marking and defense.

While we were able to prove that the proposed strategies perform efficiently for small values of the target generation rate, little is known about their performance in other regimes. In particular, we have shown numerical evidence that suggests that the first strategy we introduced, requiring no communication, performs poorly when targets are generated very frequently, whereas the performance of the sensor-based strategy is in fact comparable to that of the best known strategies for the heavy load case.

Extensions of this work will include the analysis and design of efficient strategies for general values of the target generation rate, for different vehicle dynamics models and heterogeneous systems in which both service requests and agents can belong to several different classes with different characteristics and abilities. Initial work on reactive coverage problems for multiple agents with various kinodynamic constraints has been reported in [35], [36]. Another direction of research is to extend the game theoretic formulation for the problem in this paper to formally study various hunting and foraging strategies in animals [37].

\section{ACKNOWLEDGMENT}

The authors would like to thank Dr. M. Gani for inspiring the research in this paper.

\section{REFERENCES}

[1] A. Arsie and E. Frazzoli, "Efficient routing of multiple vehicles with no explicit communications," Int. J. Robust Nonlin. Control, vol. 18, pp. 154-164, Jan. 2007.

[2] R. W. Beard, T. W. McLain, M. A. Goodrich, and E. P. Anderson, "Coordinated target assignment and intercept for unmanned air vehicles," IEEE Trans. Robot. Autom., vol. 18, no. 6, pp. 911-922, Jun. 2002.

[3] A. Richards, J. Bellingham, M. Tillerson, and J. How, "Coordination and control of multiple UAVs," in Proc. AIAA Conf. Guid., Navig., Control, Monterey, CA, 2002, pp. 1-4 [Online]. Available: http://acl.mit. edu/papers/Richards39451.pdf

[4] C. Schumacher, P. R. Chandler, S. J. Rasmussen, and D. Walker, "Task allocation for wide area search munitions with variable path length," in Proc. Amer. Control Conf., Denver, CO, 2003, pp. 3472-3477.

[5] M. Earl and R. D'Andrea, "Iterative MILP methods for vehicle control problems," IEEE Trans. Robotics, vol. 21, no. 12, pp. 1158-1167, Dec. 2005.

[6] W. Li and C. Cassandras, "A cooperative receding horizon controller for multivehicle uncertain environments," IEEE Trans. Autom. Control, vol. 51, no. 2, pp. 242-257, Feb. 2006.

[7] R. Murphey, "Target-based weapon target assignment problems," in Nonlinear Assignment Problems: Algorithms and Applications, P. Pardalos and L. Pitsoulis, Eds. Norwell, MA: Kluwer, 1999, pp. $39-53$.

[8] G. Arslan, J. R. Marden, and J. S. Shamma, "Autonomous vehicle-target assignment: A game theoretical formulation," ASME J. Dyn. Syst., Meas. Control, vol. 129, no. 5, pp. 584-596, 2007.

[9] M. Tanemura and H. Hasegawa, "Geometrical models of territory I: Models for synchronous and asynchronous settlement of territories," $J$. Theor. Biol., vol. 82, pp. 477-496, 1980.

[10] H. Psaraftis, "Dynamic vehicle routing problems," in Vehicle Routing: Methods and Studies, B. Golden and A. Assad, Eds. New York: Elsevier, 1988.

[11] D. J. Bertsimas and G. J. van Ryzin, "A stochastic and dynamic vehicle routing problem in the Euclidean plane," Oper. Res., vol. 39, pp. 601-615, 1991.

[12] E. Frazzoli and F. Bullo, "Decentralized algorithms for vehicle routing in a stochastic time-varying environment," in Proc. IEEE Conf. Decision Control, Paradise Island, Bahamas, Dec. 2004, pp. 3357-3363.

[13] Y. Liu, J. Cruz, and A. G. Sparks, "Coordinated networked uninhabited aer vehicles for persistent area denial," in Proc. IEEE Conf. Decision Control, Paradise Island, Bahamas, 2004, pp. 3351-3356.

[14] J. Cortés, S. Martínez, T. Karatas, and F. Bullo, "Coverage control for mobile sensing networks," IEEE Trans. Robot. Autom., vol. 20, no. 2, pp. 243-255, Apr. 2004.

[15] B. Moore and K. Passino, "Distributed balancing of AAVs for uniform surveillance coverage," in Proc. IEEE Conf. Decision Control, 2005, pp. 7060-7065.

[16] A. Arsie, J. J. Enright, and E. Frazzoli, "On the value of information in dynamic multiple-vehicle routing problems," in Cooperative Control of Distributed Multi-Agent Systems, J. Shamma, Ed. New York: Wiley, 2008, pp. 139-176.

[17] J. Little, "A proof of the queueing formula $l=\lambda w$," Oper. Res., vol. 9, pp. 383-387, 1961.

[18] D. J. Bertsimas and G. J. van Ryzin, "Stochastic and dynamic vehicle routing in the Euclidean plane with multiple capacitated vehicles," Oper. Res., vol. 41, no. 1, pp. 60-76, 1993.

[19] D. J. Bertsimas and G. J. van Ryzin, "Stochastic and dynamic vehicle routing with general interarrival and service time distributions," Adv. Appl. Prob., vol. 25, pp. 947-978, 1993.

[20] S. P. Lloyd, "Least squares quantization in PCM," IEEE Trans. Inform. Theory, vol. IT-28, no. 2, pp. 129-137, Mar. 1982.

[21] J. Mac Queen, L. M. LeCam and J. Neyman, Eds., "Some methods for the classification and analysis of multivariate observations," in Proc. 5th Berkeley Symp. Math. Stat. Prob, 1967, pp. 281-297.

[22] Z. Drezner, "Facility location: A survey of applications and methods," in Springer Series in Operations Research. New York: Springer Verlag, 1995.

[23] P. K. Agarwal and M. Sharir, "Efficient algorithms for geometric optimization," ACM Comput. Surveys, vol. 30, no. 4, pp. 412-458, 1998. 
[24] R. Chandrasekaran and A. Tamir, "Algebraic optimization: The Fermat-Weber location problem," Mathematical Programming, vol. 46, pp. 219-224, 1990.

[25] G. Wesolowsky, "The Weber problem: History and perspectives," Location Sci., vol. 1, pp. 5-23, 1993.

[26] S. P. Fekete, J. S. B. Mitchell, and K. Weinbrecht, "On the continuous Weber and $k$-median problems," in Proc. 16th Annu. Symp. Computat. Geom., Hong Kong, 2000, pp. 70-79.

[27] P. Carmi, S. Har-Peled, and M. Katz, "On the Fermat-Weber center of a convex object," Computat. Geom., vol. 32, no. 3, pp. 188-195, 2005.

[28] N. L. Carothers, Real Analysis. Cambridge, U.K.: Cambridge Univ. Press, 1999.

[29] M. Sabin and R. Gray, "Global convergence and empirical consistency of the generalized Lloyd algorithm," IEEE Trans. Inform. Theory, vol. 32, pp. 148-155, Mar. 1986.

[30] J. Shamma and G. Arslan, "Dynamic fictitious play, dynamic gradient play, and distributed convergence to Nash equilibria," IEEE Trans. Automat. Control, vol. 50, no. 3, pp. 312-327, Mar. 2005.

[31] D. Fudenberg and J. Tirole, Game Theory. Cambridge, MA: MIT Press, 1991

[32] R. W. Rosenthal, "A class of games possessing pure-strategy nash equilibria," Int. J. Game Theory, vol. 2, pp. 65-67, 1973.

[33] D. Monderer and L. Shapley, "Potential games," Games Econ. Beh., vol. 14, pp. 124-143, 1996.

[34] D. Wolpert and K. Tumer, Collectives and the Design of Complex Systems, ch. A Survey of Collectives. New York: Springer Verlag, 2004, p. 142 .

[35] J. Enright, K. Savla, and E. Frazzoli, "Coverage control for teams of nonholonomic agents," in Proc. IEEE Conf. Decision Control, 2008, pp. 4250-4256.

[36] K. Savla and E. Frazzoli, "On endogenous reconfiguration for mobile robotic networks," in Proc. Workshop Algorithmic Found. Robot. (WAFR), Guanajuato, Mexico, Dec. 2008.

[37] L. A. Dugatkin and H. K. Reeve, Game Theory and Animal Behavior. Oxford, U.K.: Oxford Univ. Press, 2000.

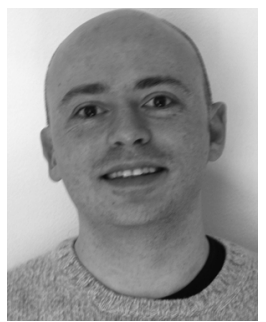

Alessandro Arsie received the B.Sc. degree in physics from the University of Padova, Padova, Italy in 1997, the Ph.D. degree in mathematical physics from the International School for Advanced Studies, Trieste, Italy, in 2001, and the M.Sc. degree in quantitative finance and risk management from the Business University Bocconi, Milan, Italy, in 2005.

He has been a Postdoctoral Associate in the Department of Mathematics, University of Bologna, Bologna, Italy, a Postdoctoral Associate of Mechanical and Aerospace Engineering at UCLA, in the Laboratory for Information and Decision Systems, Massachusetts Institute of Technology, Cambridge, and he is currently a Postdoctoral Associate in the Department of Mathematics, Penn State University, University Park. His past research interests were focused on algebraic geometry while his current research interests include decentralized control of multi-agent systems, control of impulsive Lagrangian systems, and geometric methods in control and optimization.

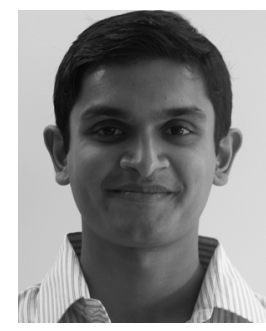

Ketan Savla (S '06) received the B.Tech. degree in mechanical engineering from the Indian Institute of Technology, Bombay, in 2003, the M.S. degree in mechanical engineering from the University of Illinois at Urbana-Champaign in 2004, and the M.A. degree in applied mathematics and the Ph.D. degree in electrical engineering from the University of California at Santa Barbara in 2007.

$\mathrm{He}$ is currently a Postdoctoral Associate in the Laboratory for Information and Decision Systems, Massachusetts Institute of Technology, Cambridge. His current research interests include algorithmic mobile robotics, coordination of multiple autonomous vehicles, and humans-in-the-loop control systems.

Dr. Savlas CDC-ECC05 paper was a Finalist for the Best Student Paper Award.

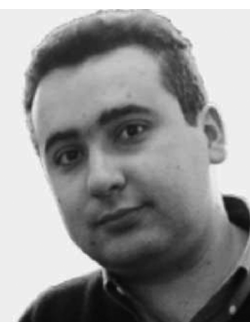

Emilio Frazzoli (S'99-M'01-SM'08) received the Laurea degree in aerospace engineering from the University of Rome La Sapienza, Rome, Italy, in 1994 and the Ph.D. degree in navigation and contro systems from the Department of Aeronautics and Astronautics, Massachusetts Institute of Technology (MIT), Cambridge, in 2001.

From 2001 to 2004, he was an Assistant Professor of aerospace engineering at the University of Illinois at Urbana-Champaign. From 2004 to 2006, he was an Assistant Professor of mechanical and aerospace engineering at the University of California, Los Angeles. He is currently an Associate Professor of aeronautics and astronautics with the Laboratory for Information and Decision Systems, Department of Aeronautics and Astronautics, MIT. His current research interests include algorithmic, computational, and geometric methods for the design of complex control systems, in aerospace and other domains, and the application areas include distributed cooperative control of multiple-vehicle systems, guidance and control of agile vehicles, mobile robotics, and high-confidence embedded systems.

Dr. Frazzoli received the National Science Foundation (NSF) CAREER Award in 2002. 